\title{
K-Semistability of cscK Manifolds with Transcendental Cohomology Class
}

\author{
Zakarias Sjöström Dyrefelt ${ }^{1,2}$
}

Received: 24 February 2017 / Published online: 16 October 2017

(C) The Author(s) 2017

\begin{abstract}
We prove that constant scalar curvature Kähler (cscK) manifolds with transcendental cohomology class are K-semistable, naturally generalising the situation for polarised manifolds. Relying on a recent result by R. Berman, T. Darvas and C. Lu regarding properness of the K-energy, it moreover follows that cscK manifolds with discrete automorphism group are uniformly K-stable. As a main step of the proof we establish, in the general Kähler setting, a formula relating the (generalised) DonaldsonFutaki invariant to the asymptotic slope of the K-energy along weak geodesic rays.
\end{abstract}

Keywords Constant scalar curvature Kähler metric $\cdot$ K-Stability $\cdot$ Energy functional asymptotics · YTD conjecture

Mathematics Subject Classification 32Q26 · 32Q15 $\cdot$ 14D06 $\cdot$ 53C56 $\cdot$ 14F43

\section{Introduction}

In this paper we are interested in questions of stability for constant scalar curvature Kähler (cscK) manifolds with transcendental ${ }^{1}$ cohomology class. To this end, let $(X, \omega)$ be a compact Kähler manifold and $\alpha:=[\omega] \in H^{1,1}(X, \mathbb{R})$ the corresponding Kähler class. When $\alpha$ is the first Chern class $c_{1}(L)$ of some ample line bundle $L$ over

\footnotetext{
1 We use the word 'transcendental' to emphasise that the Kähler class in question is not necessarily of the form $c_{1}(L)$ for some ample line bundle $L$ over $X$.

Z Zakarias Sjöström Dyrefelt zakarias.sjostrom@math.univ-toulouse.fr

1 Institut de Mathématiques de Toulouse, Université Paul Sabatier, 118 route de Narbonne, 31062 Toulouse Cedex 9, France

2 Centre de Mathématiques Laurent Schwartz, École Polytechnique, 91128 Palaiseau Cedex, France
} 
$X$, such questions are closely related to the Yau-Tian-Donaldson (YTD) conjecture [27,49,54]: A polarised algebraic manifold $(X, L)$ is K-polystable if and only if the polarisation class $c_{1}(L)$ admits a Kähler metric of constant scalar curvature. This conjecture was recently confirmed in the Fano case, i.e. when $L=-K_{X}$, cf. [16$18,52]$. In this important special case, a cscK metric is nothing but a Kähler-Einstein metric. For general polarised cscK manifolds, the "if" direction of the YTD conjecture was initially proven by Mabuchi in [37], see also [5]. Prior to that, several partial results had been obtained by Donaldson [28] and Stoppa [46], both assuming that $c_{1}(L)$ contains a cscK metric.

For transcendental classes very little is currently known about the validity of a correspondence between existence of cscK metrics and stability in the spirit of the YTD conjecture. Moreover, from a differential geometric point of view, there is no special reason to restrict attention to Kähler manifolds with associated integral (or rational) cohomology classes, which are then automatically of the form $\alpha=c_{1}(L)$ for some ample $(\mathbb{Q})$-line bundle $L$ over $X$. In order to extend the study of stability questions to a transcendental setting, recall that there is an intersection theoretic description of the Donaldson-Futaki invariant, cf. [39,53]. As first pointed out by Berman [4], a straightforward generalised notion of K-stability in terms of cohomology can thus be defined and a version of the YTD conjecture can be made sense of in this setting. The setup is explained in detail in Sect. 3. Our main goal is to establish the following result:

Theorem A Let $(X, \omega)$ be a compact Kähler manifold and let $\alpha:=[\omega] \in H^{1,1}(X, \mathbb{R})$ be the corresponding Kähler class.

(i) If the Mabuchi (K-energy) functional is bounded from below in $\alpha$, then $(X, \alpha)$ is $\mathrm{K}$-semistable (in the generalised sense of Definition 3.8).

(ii) If the Mabuchi functional is coercive, then $(X, \alpha)$ is uniformly K-stable the generalised sense of Definition 5.4).

For precise definitions we refer to the core of the paper. As an immediate consequence of [6, Theorem 1.1] and the above Theorem A (i) we obtain the following corollary, which is a main motivation for our work (see also Remark 1.2).

Corollary 1.1 If the Kähler class $\alpha \in H^{1,1}(X, \mathbb{R})$ admits a constant scalar curvature representative, then $(X, \alpha)$ is $K$-semistable.

The corresponding statement in the case of a polarised manifold was first obtained by Donaldson in [28], as an immediate consequence of the lower bound for the Calabi functional. See also [43,47] for related work on slope semistability. The approach taken in this paper should however be compared to, e.g. [42] and [4,5,12,13], where $\mathrm{K}$-semistability is derived using so called "Kempf-Ness type" formulas. By analogy to the above papers, our proof relies on establishing such formulas valid also for transcendental classes (see Theorems B and C), in particular relating the asymptotic slope of the K-energy along weak geodesic rays to a natural generalisation of the Donaldson-Futaki invariant. This provides a link between K-semistability (resp. uniform K-stability) and boundedness (resp. coercivity) of the Mabuchi functional, key to establishing the stability results of Theorem A. 
An underlying theme of the paper is the comparison to the extensively studied case of a polarised manifold, which becomes a "special case" in our setting. Notably, it is then known (see, e.g. $[4,5,12,13]$ ) how to establish the sought Kempf-Ness type formulas using Deligne pairings; a method employed by Phong-Ross-Sturm in [42] (for further background on the Deligne pairing construction, cf. [30]). Unfortunately, such an approach breaks down in the case of a general Kähler class. In this paper, we circumvent this problem by a pluripotential approach, making use of a certain multivariate variant $\left\langle\varphi_{0}, \ldots, \varphi_{n}\right\rangle_{\left(\theta_{0}, \ldots \theta_{n}\right)}$ of the Monge-Ampère energy functional, which turns out to play a role analogous to that of the Deligne pairing in arguments of the type [42]. The Deligne pairing approach should also be compared to $[26,50]$ using Bott-Chern forms (see, e.g. 2.4 and [44, Example 5.6]).

Remark 1.2 (Yau-Tian-Donaldson conjecture) Combining Theorem A (ii) with [22, Theorem 2.10] and a very recent result by Berman et al. [8, Theorem 1.2] we in fact further see that $\operatorname{cscK}$ manifolds $(X, \alpha)$ with discrete automorphism group are uniformly K-stable. The above thus confirms one direction of the Yau-Tian-Donaldson conjecture, here referring to its natural generalisation to the case of arbitrary compact Kähler manifolds with discrete automorphism group, see Sect. 5.2.

\subsection{Generalised K-Semistability}

We briefly explain the framework we have in mind. As a starting point, there are natural generalisations of certain key concepts to the transcendental setting, a central notion being that of test configurations. First recall that a test configuration for a polarised manifold $(X, L)$, in the sense of Donaldson, cf. [27], is given in terms of a $\mathbb{C}^{*}$-equivariant degeneration $(\mathcal{X}, \mathcal{L})$ of $(X, L)$. It can be seen as an algebrogeometric way of compactifying the product $X \times \mathbb{C}^{*} \hookrightarrow \mathcal{X}$. Note that test configurations in the sense of Donaldson are now known (at least in the case of Fano manifolds, see [36]) to be equivalent to test configurations in the sense of Tian [49].

As remarked in [4], a straightforward generalisation to the transcendental setting can be given by replacing the line bundles with $(1,1)$-cohomology classes. In the polarised setting we would thus consider $\left(\mathcal{X}, c_{1}(\mathcal{L})\right)$ as a "test configuration" for $\left(X, c_{1}(L)\right)$, by simply replacing $\mathcal{L}$ and $L$ with their respective first Chern classes. The details of how to formulate a good definition of such a generalised test configuration have, however, not yet been completely clarified. The definition given in this paper is motivated by a careful comparison to the usual polarised case, where we ensure that a number of basic but convenient tools still hold, cf. Sect. 3. In particular, our notion of K-semistability coincides precisely with the usual one whenever we restrict to the case of an integral class, cf. Proposition 3.14. We will refer to such generalised test configurations as cohomological.

Definition 1.3 (Cohomological test configuration) A cohomological test configuration for $(X, \alpha)$ is a pair $(\mathcal{X}, \mathcal{A})$ where $\mathcal{X}$ is a test configuration for $X$ (see Definition 3.2) and $\mathcal{A} \in H_{\mathrm{BC}}^{1,1}(\mathcal{X}, \mathbb{R})^{\mathbb{C}^{*}}$ is a $\mathbb{C}^{*}$-invariant $(1,1)$-Bott-Chern cohomology class whose image under the canonical $\mathbb{C}^{*}$-equivariant isomorphism 


$$
\mathcal{X} \backslash \mathcal{X}_{0} \simeq X \times\left(\mathbb{P}^{1} \backslash\{0\}\right)
$$

is $p_{1}^{*} \alpha$, see (6). Here $p_{1}: X \times \mathbb{P}^{1} \rightarrow X$ denotes the first projection.

Remark 1.4 Note that the definition is given directly over $\mathbb{P}^{1}$ so that we consider the Bott-Chern cohomology on a compact Kähler normal complex space. In the polarised case, defining a test configuration over $\mathbb{C}$ or over $\mathbb{P}^{1}$ is indeed equivalent, due to the existence of a natural $\mathbb{C}^{*}$-equivariant compactification over $\mathbb{P}^{1}$.

In practice, it will be enough to consider the situation when the total space $\mathcal{X}$ is smooth and dominates $X \times \mathbb{P}^{1}$, with $\mu: \mathcal{X} \rightarrow X \times \mathbb{P}^{1}$ the corresponding canonical $\mathbb{C}^{*}$-equivariant bimeromorphic morphism. Moreover, if $(\mathcal{X}, \mathcal{A})$ is a cohomological test configuration for $(X, \alpha)$ with $\mathcal{X}$ as above, then $\mathcal{A}$ is always of the form $\mathcal{A}=\mu^{*} p_{1}^{*} \alpha+[D]$, for a unique $\mathbb{R}$-divisor $D$ supported on the central fibre $\mathcal{X}_{0}$, cf. Proposition 3.10. A cohomological test configuration can thus be characterised by an $\mathbb{R}$-divisor, clarifying the relationship between the point of view of $\mathbb{R}$-divisors and our cohomological approach to "transcendental K-semistability".

A straightforward generalisation of the Donaldson-Futaki invariant can be defined based on the intersection theoretic characterisation of $[39,53]$. Indeed, we define the Donaldson-Futaki invariant associated with a cohomological test configuration $(\mathcal{X}, \mathcal{A})$ for $(X, \alpha)$ as the following intersection number

$$
\operatorname{DF}(\mathcal{X}, \mathcal{A}):=\frac{\overline{\mathcal{S}}}{n+1} V^{-1}\left(\mathcal{A}^{n+1}\right) \mathcal{X}+V^{-1}\left(K_{\mathcal{X} / \mathbb{P}^{1}} \cdot \mathcal{A}^{n}\right) \mathcal{X},
$$

computed on the (compact) total space $\mathcal{X}$. Here $V$ and $\overline{\mathcal{S}}$ are cohomological constants denoting the Kähler volume and mean scalar curvature of $(X, \alpha)$, respectively.

Finally, we say that $(X, \alpha)$ is $K$-semistable if $\operatorname{DF}(\mathcal{X}, \mathcal{A}) \geqslant 0$ for all cohomological test configurations $(\mathcal{X}, \mathcal{A})$ for $(X, \alpha)$ where the class $\mathcal{A}$ is relatively Kähler, i.e. there is a Kähler form $\beta$ on $\mathbb{P}^{1}$ such that $\mathcal{A}+\pi^{*} \beta$ is Kähler on $\mathcal{X}$. Generalised notions of (uniform) K-stability are defined analogously.

\subsection{Transcendental Kempf-Ness Type Formulas}

As previously stated, a central part of this paper consists in establishing a KempfNess type formula connecting the Donaldson-Futaki invariant (in the sense of (1)) with the asymptotic slope of the K-energy along certain weak geodesic rays. In fact, we first prove the following result, which is concerned with asymptotics of a certain multivariate analogue of the Monge-Ampère energy, cf. Sect. 2.2 for its definition. It turns out to be very useful for establishing a similar formula for the K-energy (cf. Remark 1.5), but may also be of independent interest.

In what follows, we will work on the level of potentials and refer the reader to Sect. 4 for precise definitions.

Theorem $\mathbf{B}$ Let $X$ be a compact Kähler manifold of dimension $n$ and let $\theta_{i}, 0 \leqslant i \leqslant n$, be closed $(1,1)$-forms on $X$. Let $\left(\mathcal{X}_{i}, \mathcal{A}_{i}\right)$ be cohomological test configurations for 
$\left(X, \alpha_{i}\right)$, where $\alpha_{i}:=\left[\theta_{i}\right] \in H^{1,1}(X, \mathbb{R})$. Then, for each collection of smooth rays $\left(\varphi_{i}^{t}\right)_{t \geqslant 0}, \mathcal{C}^{\infty}$-compatible with $\left(\mathcal{X}_{i}, \mathcal{A}_{i}\right)$, respectively, the asymptotic slope of the multivariate energy functional $\langle\cdot, \ldots, \cdot\rangle:=\langle\cdot, \ldots, \cdot\rangle_{\left(\theta_{0}, \ldots, \theta_{n}\right)}$ is well defined and satisfies

$$
\frac{\left\langle\varphi_{0}^{t}, \ldots, \varphi_{n}^{t}\right\rangle}{t} \longrightarrow\left(\mathcal{A}_{0} \cdots \mathcal{A}_{n}\right)
$$

as $t \rightarrow+\infty$. See Sect. 4.1 for the definition of the above intersection number.

Remark 1.5 In the setting of Hermitian line bundles, the above multivariate energy functional naturally appears as the difference (or quotient) of metrics on Deligne pairings. Moreover, note that the above theorem applies to, e.g. Aubin's J-functional, the Monge-Ampère energy functional $\mathrm{E}$ and its 'twisted' version $\mathrm{E}^{\mathrm{Ric}(\omega)}$ but not to the K-energy M. Indeed, the expression for $\mathbf{M}\left(\varphi_{t}\right)$ on the form $\left\langle\varphi_{0}^{t}, \ldots, \varphi_{n}^{t}\right\rangle_{\left(\theta_{0}, \ldots, \theta_{n}\right)}$ involves the metric $\log \left(\omega+d d^{c} \varphi_{t}\right)^{n}$ on the relative canonical bundle $K_{\mathcal{X} / \mathbb{P}^{1}}$, which blows up close to $\mathcal{X}_{0}$, cf. Sect. 5. As observed in [13], it is however possible to find functionals of the above form that 'approximate' $M$ in the sense that their asymptotic slopes coincide, up to an explicit correction term that vanishes precisely when the central fibre $\mathcal{X}_{0}$ is reduced. This is a key observation.

We further remark that such a formula (2) cannot be expected to hold unless the test configurations $\left(\mathcal{X}_{i}, \mathcal{A}_{i}\right)$ and the rays $\left(\varphi_{i}^{t}\right)$ are compatible in a certain sense. This is the role of the notion of $\mathcal{C}^{\infty}$-compatibility (as well as the $\mathcal{C}^{1, \overline{1}}$-compatibility used in Theorem C). These notions may seem technical, but in fact mimic the case of a polarised manifold, where the situation is well understood in terms of extension of metrics on line bundles, cf. Sect. 4.1.

As a further important consequence of the above Theorem $\mathrm{B}$ we deduce that if $(\mathcal{X}, \mathcal{A})$ is a relatively Kähler cohomological test configuration for $(X, \alpha)$, then for each smooth ray $\left(\varphi_{t}\right)_{t \geqslant 0}, \mathcal{C}^{\infty}$-compatible with $(\mathcal{X}, \mathcal{A})$, we have the inequality

$$
\lim _{t \rightarrow+\infty} \frac{\mathrm{M}\left(\varphi_{t}\right)}{t} \leqslant \operatorname{DF}(\mathcal{X}, \mathcal{A})
$$

This is the content of Theorem 5.1, and should be compared to the discussion in the introduction of [42]. As an important special case, this inequality can be seen to hold in the case of a weak geodesic ray associated with the given test configuration $(\mathcal{X}, \mathcal{A})$, cf. Sect. 4.1 for its construction. The inequality (3) is moreover enough to conclude the proof of Theorem A, as explained in Sect. 5.2.

Using ideas from [13] adapted to the present setting, we may further improve on formula (3) and compute the precise asymptotic slope of the K-energy. In this context, it is natural to consider the non-Archimedean Mabuchi functional

$$
\mathrm{M}^{\mathrm{NA}}(\mathcal{X}, \mathcal{A}):=\operatorname{DF}(\mathcal{X}, \mathcal{A})+V^{-1}\left(\left(\mathcal{X}_{0, \text { red }}-\mathcal{X}_{0}\right) \cdot \mathcal{A}^{n}\right) \mathcal{X}
$$

cf. $[12,13]$ for an explanation of the terminology. It is a modification of the DonaldsonFutaki invariant which is homogeneous under finite base change, and which satisfies 
$\mathrm{M}^{\mathrm{NA}}(\mathcal{X}, \mathcal{A}) \leqslant \operatorname{DF}(\mathcal{X}, \mathcal{A})$ with equality precisely when the central fibre $\mathcal{X}_{0}$ is reduced. We then have the following result, special cases of which have been obtained by previous authors in various different situations and generality.

Theorem $\mathbf{C}$ Let $(\mathcal{X}, \mathcal{A})$ be a smooth, relatively Kähler cohomological test configuration for $(X, \alpha)$ dominating $X \times \mathbb{P}^{1}$. For each subgeodesic ray $\left(\varphi_{t}\right)_{t \geqslant 0}, \mathcal{C}^{1, \overline{1}}$-compatible with $(\mathcal{X}, \mathcal{A})$, the following limit is well defined and satisfies

$$
\frac{\mathrm{M}\left(\varphi_{t}\right)}{t} \longrightarrow \mathrm{M}^{\mathrm{NA}}(\mathcal{X}, \mathcal{A})
$$

as $t \rightarrow+\infty$. In particular, this result holds for the weak geodesic ray associated with $(\mathcal{X}, \mathcal{A})$, constructed in Lemma 4.6.

Remark 1.6 When the class $\mathcal{A}$ on $\mathcal{X}$ is merely relatively nef it is possible to obtain similar statements, but this necessitates much more involved arguments. Either way, the above result is more than enough for our purposes here, e.g. for proving the main result, Theorem A.

For polarised manifolds $(X, L)$ and smooth subgeodesic rays $\left(\varphi_{t}\right)_{t \geqslant 0}$, this precise result was proven in [13] using Deligne pairings, as pioneered by Phong-Ross-Sturm in [42] (cf. also Paul-Tian [40,41]). A formula in the same spirit has also been obtained for the so- called Ding functional when $X$ is a Fano variety, see [5]. However, it appears as though no version of this result was previously known in the case of non-polarised manifolds.

\subsection{Structure of the Paper}

In Sect. 2 we fix our notation for energy functionals and subgeodesic rays. In particular, we introduce the multivariate energy functionals $\langle\cdot, \ldots, \cdot\rangle_{\left(\theta_{0}, \ldots, \theta_{n}\right)}$, which play a central role in this paper. In Sect. 3 we introduce our generalised notion of cohomological test configurations and K-semistability. In the case of a polarised manifold $(X, L)$, we compare this notion to the usual algebraic one. We also discuss classes of cohomological test configurations for which it suffices to test K-semistability, and establish a number of basic properties. In Sect. 4 we discuss transcendental Kempf-Ness-type formulas and prove Theorem $\mathrm{B}$. This involves introducing natural compatibility conditions between a ray $\left(\varphi_{t}\right)$ and a cohomological test configuration $(\mathcal{X}, \mathcal{A})$ for $(X, \alpha)$. As a useful special case, we discuss the weak geodesic ray associated with $(\mathcal{X}, \mathcal{A})$. In Sect. 5 we finally apply Theorem B to yield a weak version of Theorem $C$, from which we in turn deduce our main result, Theorem A. By an immediate adaptation of techniques from [13] we then compute the precise asymptotic slope of the Mabuchi functional, thus establishing the full Theorem $\mathrm{C}$. 


\section{Preliminaries}

\subsection{Notation and Basic Definitions}

Let $X$ be a compact complex manifold of $\operatorname{dim}_{\mathbb{C}} X=n$ equipped with a given Kähler form $\omega$, i.e. a smooth real closed positive $(1,1)$-form on $X$. Denote the Kähler class $[\omega] \in H^{1,1}(X, \mathbb{R})$ by $\alpha$.

In order to fix notation, let $\operatorname{Ric}(\omega)=-d d^{c} \log \omega^{n}$ be the Ricci curvature form, where $d d^{c}:=\frac{\sqrt{-1}}{2 \pi} \partial \bar{\partial}$ is normalised so that $\operatorname{Ric}(\omega)$ represents the first Chern class $c_{1}(X)$. Its trace

$$
\mathcal{S}(\omega):=n \frac{\operatorname{Ric}(\omega) \wedge \omega^{n-1}}{\omega^{n}}
$$

is the scalar curvature of $\omega$. The mean scalar curvature is the cohomological constant given by

$$
\overline{\mathcal{S}}:=V^{-1} \int_{X} \mathcal{S}(\omega) \omega^{n}=n \frac{\int_{X} c_{1}(X) \cdot \alpha^{n-1}}{\int_{X} \alpha^{n}}:=n \frac{\left(c_{1}(X) \cdot \alpha^{n-1}\right)_{X}}{\left(\alpha^{n}\right)_{X}},
$$

where $V:=\int_{X} \omega^{n}:=\left(\alpha^{n}\right)_{X}$ is the Kähler volume. We say that $\omega$ is a constant scalar curvature Kähler (cscK) metric ${ }^{2}$ if $S(\omega)$ is constant (equal to $\overline{\mathcal{S}}$ ) on X.

Throughout the paper we work on the level of potentials, using the notation of quasi-plurisubharmonic (quasi-psh) functions. To this end, we let $\theta$ be a closed $(1,1)$ form on $X$ and denote, as usual, by $\operatorname{PSH}(X, \theta)$ the space of $\theta$-psh functions $\varphi$ on $X$, i.e. the set of functions that can be locally written as the sum of a smooth and a plurisubharmonic function, and such that

$$
\theta_{\varphi}:=\theta+d d^{c} \varphi \geqslant 0
$$

in the weak sense of currents. In particular, if $\omega$ is our fixed Kähler form on $X$, then we write

$$
\mathcal{H}_{\omega}:=\left\{\varphi \in \mathcal{C}^{\infty}(X): \omega_{\varphi}:=\omega+d d^{c} \varphi>0\right\} \subset \operatorname{PSH}(X, \omega)
$$

for the space of Kähler potentials on $X$. As a subset of $\mathcal{C}^{\infty}(X)$ it is convex and consists of strictly $\omega$-psh functions. It has been extensively studied (for background we refer the reader to, e.g. [10] and references therein).

Recall that a $\theta$-psh function is always upper semi-continuous (usc) on $X$, thus bounded from above by compactness. Moreover, if $\varphi_{i} \in \operatorname{PSH}(X, \theta) \cap L_{\text {loc }}^{\infty}, 1 \leqslant i \leqslant$ $p \leqslant n$, it follows from the work of Bedford-Taylor [2,3] that we can give meaning to the product $\bigwedge_{i=1}^{p}\left(\theta+d d^{c} \varphi_{i}\right)$, which then defines a closed positive $(p, p)$-current on $X$. As usual, we then define the Monge-Ampère measure as the following probability measure, given by the top wedge product

\footnotetext{
2 By a standard abuse of notation we identify a Hermitian metric $h$ with its associated $(1,1)$-form $\omega=\omega_{h}$ and refer to $\omega$ as the "metric".
} 


$$
\operatorname{MA}(\varphi):=V^{-1}\left(\omega+d d^{c} \varphi\right)^{n}
$$

\subsection{Energy Functionals and a Deligne Functional Formalism}

We now introduce the notation for energy functionals that we will use. Let $\varphi_{i} \in$ $\operatorname{PSH}(X, \omega) \cap L_{\mathrm{loc}}^{\infty}$. The Monge-Ampère energy functional (or Aubin-Mabuchi functional) $\mathrm{E}:=\mathrm{E}_{\omega}$ is defined by

$$
\mathrm{E}(\varphi):=\frac{1}{n+1} \sum_{j=0}^{n} V^{-1} \int_{X} \varphi\left(\omega+d d^{c} \varphi\right)^{n-j} \wedge \omega^{j} .
$$

Similarly, if $\theta$ is any closed $(1,1)$-form, we define a functional $\mathrm{E}^{\theta}:=\mathrm{E}_{\omega}^{\theta}$ by

$$
\mathrm{E}^{\theta}(\varphi):=\sum_{j=0}^{n-1} V^{-1} \int_{X} \varphi\left(\omega+d d^{c} \varphi\right)^{n-j-1} \wedge \omega^{j} \wedge \theta
$$

and we will also have use for the Aubin J-functional J : PSH $(X, \omega) \cap L_{\text {loc }}^{\infty} \rightarrow \mathbb{R}_{\geqslant 0}$ defined by

$$
\mathrm{J}(\varphi):=V^{-1} \int_{X} \varphi \omega^{n}-\mathrm{E}(\varphi)
$$

whose asymptotic slope along geodesic rays is comparable with the minimum norm of a test configuration (see $[12,24]$ ).

More generally, it is possible to define a natural multivariate version of the Monge-Ampère energy, of which all of the above functionals are special cases. The construction builds on that of the Deligne pairing, which is a powerful and general technique from algebraic geometry that we here apply to our specific setting in Kähler geometry. We refer the interested reader to $[30,38,55]$ for a general treatment of Deligne pairings, as well as to $[5,13,42]$ for more recent applications related to Kstability. Now let $\theta_{0}, \ldots, \theta_{n}$ be closed $(1,1)$-forms on $X$. Motivated by corresponding properties for the Deligne pairing (cf., e.g. [5,30] for background) we would like to consider a functional $\langle\cdot, \ldots, \cdot\rangle_{\left(\theta_{0}, \ldots, \theta_{n}\right)}$ on the space $\operatorname{PSH}\left(X, \theta_{0}\right) \cap L_{\mathrm{loc}}^{\infty} \times \cdots \times$ $\operatorname{PSH}\left(X, \theta_{n}\right) \cap L_{\text {loc }}^{\infty}(n+1$ times $)$ that is

- symmetric, i.e. for any permutation $\sigma$ of the set $\{0,1, \ldots, n\}$, we have

$$
\left\langle\varphi_{\sigma(0)}, \ldots, \varphi_{\sigma(n)}\right\rangle_{\left(\theta_{\sigma(0)}, \ldots, \theta_{\sigma(n)}\right)}=\left\langle\varphi_{0}, \ldots, \varphi_{n}\right\rangle_{\left(\theta_{0}, \ldots, \theta_{n}\right)}
$$

- if $\varphi_{0}^{\prime}$ is another $\theta_{i}$-psh function in $\operatorname{PSH}(X, \theta) \cap L_{\text {loc }}^{\infty}$, then we have a 'change of function' property

$$
\begin{aligned}
& \left\langle\varphi_{0}^{\prime}, \varphi_{1} \ldots, \varphi_{n}\right\rangle-\left\langle\varphi_{0}, \varphi_{1} \ldots, \varphi_{n}\right\rangle \\
& \quad=\int_{X}\left(\varphi_{0}^{\prime}-\varphi_{0}\right)\left(\omega_{1}+d d^{c} \varphi_{1}\right) \wedge \cdots \wedge\left(\omega_{n}+d d^{c} \varphi_{n}\right) .
\end{aligned}
$$


Demanding that the above properties hold necessarily leads to the following definition of Deligne functionals, that will provide a useful terminology for this paper.

Definition 2.1 Let $\theta_{0}, \ldots, \theta_{n}$ be closed $(1,1)$-forms on $X$. Define a multivariate energy functional $\langle\cdot, \ldots, \cdot\rangle_{\left(\theta_{0}, \ldots, \theta_{n}\right)}$ on the space $\operatorname{PSH}\left(X, \theta_{0}\right) \cap L_{\mathrm{loc}}^{\infty} \times \cdots \times$ $\operatorname{PSH}\left(X, \theta_{n}\right) \cap L_{\text {loc }}^{\infty}(n+1$ times $)$ by

$$
\begin{aligned}
& \left\langle\varphi_{0}, \ldots, \varphi_{n}\right\rangle_{\left(\theta_{0}, \ldots, \theta_{n}\right)}:=\int_{X} \varphi_{0}\left(\theta_{1}+d d^{c} \varphi_{1}\right) \wedge \cdots \wedge\left(\theta_{n}+d d^{c} \varphi_{n}\right) \\
& \quad+\int_{X} \varphi_{1} \theta_{0} \wedge\left(\theta_{2}+d d^{c} \varphi_{2}\right) \wedge \cdots \wedge\left(\theta+d d^{c} \varphi_{n}\right)+\cdots+\int_{X} \varphi_{n} \theta_{0} \wedge \cdots \wedge \theta_{n-1} .
\end{aligned}
$$

Remark 2.2 The multivariate energy functional $\langle\cdot, \ldots, \cdot\rangle_{\left(\theta_{0}, \ldots, \theta_{n}\right)}$ can also be defined on $\mathcal{C}^{\infty}(X) \times \cdots \times \mathcal{C}^{\infty}(X)$ by the same formula. In Sects. 4 and 5 it will be interesting to consider both the smooth case and the case of locally bounded $\theta_{i}$-psh functions.

Using integration by parts one can check that this functional is indeed symmetric.

Proposition 2.3 The functional $\langle\cdot, \ldots, \cdot\rangle_{\left(\theta_{0}, \ldots, \theta_{n}\right)}$ is symmetric.

Proof Since every permutation is a composition of transpositions it suffices to check the sought symmetry property for transpositions $\sigma:=\sigma_{j, k}$ exchanging the position of $j, k \in\{0,1, \ldots, n\}$. Suppose for simplicity of notation that $j<k$ and write $\theta_{i}^{t}:=\theta_{i}+d d^{c} \varphi_{i}$. A straightforward computation then yields

$$
\begin{aligned}
& \left\langle\varphi_{0}, \ldots, \varphi_{j}, \varphi_{k}, \ldots \varphi_{n}\right\rangle_{\left(\theta_{0}, \ldots, \theta_{j}, \theta_{k}, \ldots \theta_{n}\right)}-\left\langle\varphi_{0}, \ldots, \varphi_{k}, \varphi_{j}, \ldots \varphi_{n}\right\rangle_{\left(\theta_{0}, \ldots, \theta_{k}, \theta_{j}, \ldots \theta_{n}\right)} \\
& =\int_{X} \varphi_{j} d d^{c} \varphi_{k} \wedge \Theta_{j, k}-\int_{X} \varphi_{k} d d^{c} \varphi_{j} \wedge \Theta_{j, k}=0
\end{aligned}
$$

where in the last step we used integration by parts and write

$$
\Theta_{j, k}:=\theta_{0} \wedge \cdots \wedge \theta_{j-1} \wedge \theta_{j+1}^{t} \wedge \ldots \theta_{k-1}^{t} \wedge \theta_{k+1}^{t} \wedge \theta_{n}^{t}
$$

(with factors $\theta_{j}$ and $\theta_{k}^{t}$ omitted). The case $j>k$ follows in the exact same way, with obvious modifications to the above proof.

Example 2.4 As previously remarked, note that the above functionals can be written using the Deligne functional formalism. Indeed, if $\theta$ is a closed $(1,1)$-form on $X, \omega$ is a Kähler form on $X$ and $\varphi$ is an $\omega$-psh function on $X$, then

$$
\mathrm{E}(\varphi)=\frac{1}{n+1} V^{-1}\langle\varphi, \ldots, \varphi\rangle_{(\omega, \ldots, \omega)}, \quad \mathrm{E}^{\theta}(\varphi)=V^{-1}\langle 0, \varphi, \ldots, \varphi\rangle_{(\theta, \omega, \ldots, \omega)}
$$

and

$$
\mathrm{J}(\varphi)=V^{-1}\langle\varphi, 0, \ldots, 0\rangle_{(\omega, \ldots, \omega)}-\mathrm{E}(\varphi) .
$$

Compare also [44, Example 5.6] on Bott-Chern forms. 


\subsection{Subgeodesic Rays}

Let $\left(\varphi_{t}\right)_{t \geqslant 0} \subset \operatorname{PSH}(X, \omega)$ be a ray of $\omega$-psh functions. Following a useful point of view of Donaldson [27] and Semmes [45], there is a basic correspondence between the family $\left(\varphi_{t}\right)_{t \geqslant 0}$ and an associated $S^{1}$-invariant function $\Phi$ on $X \times \bar{\Delta}^{*}$, where $\bar{\Delta}^{*} \subset \mathbb{C}$ denotes the punctured unit disc. We denote by $\tau$ the coordinate on $\Delta$. Explicitly, the correspondence is given by

$$
\Phi\left(x, e^{-t+i s}\right)=\varphi^{t}(x),
$$

where the sign is chosen so that $t \rightarrow+\infty$ corresponds to $\tau:=e^{-t+i s} \rightarrow 0$. The function $\Phi$ restricted to a fibre $X \times\{\tau\}$ thus corresponds precisely to $\varphi_{t}$ on $X$. In the direction of the fibres we thus have $p_{1}^{*} \omega+d d_{x}^{c} \Phi \geqslant 0$ (in the sense of currents, letting $p_{1}: X \times \Delta \rightarrow X$ denote the first projection).

We will use the following standard terminology, motivated by the extensive study of (weak) geodesics in the space $\mathcal{H}$, see, e.g. [9, 15,20,27,45].

Definition 2.5 We say that $\left(\varphi_{t}\right)_{t \geqslant 0}$ is a subgeodesic ray if the associated $S^{1}$-invariant function $\Phi$ on $X \times \bar{\Delta}^{*}$ is $p_{1}^{*} \omega$-psh. Furthermore, a locally bounded family of functions $\left(\varphi_{t}\right)_{t \geqslant 0}$ in $\operatorname{PSH}(X, \omega)$ is said to be a weak geodesic ray if the associated $S^{1}$-invariant function $\Phi \in \operatorname{PSH}\left(X \times \bar{\Delta}^{*}, p_{1}^{*} \omega\right)$ satisfies

$$
\left(p_{1}^{*} \omega+d d_{(x, \tau)}^{c} \Phi\right)^{n+1}=0
$$

on $X \times \Delta^{*}$.

Definition 2.6 Viewing the family $\left(\varphi_{t}\right)_{t \geqslant 0}$ as a map $(0,+\infty) \rightarrow \operatorname{PSH}(X, \omega)$, we say that $\left(\varphi^{t}\right)_{t \geqslant 0}$ is continuous (resp. locally bounded, smooth) if the corresponding $S^{1}$-invariant function $\Phi$ is continuous (resp. locally bounded, smooth).

The existence of geodesics with bounded Laplacian was proven by Chen [15] with complements by Blocki [9], see also, e.g. [20,21]. We will refer to such a geodesic as being $\mathcal{C}^{1, \overline{1}}$-regular, cf. Lemma 4.6 .

Definition 2.7 We say that a function $\varphi$ is $\mathcal{C}^{1, \overline{1}}$-regular if $d d^{c} \varphi \in L_{\text {loc }}^{\infty}$, and we set $\mathcal{H}^{1, \overline{1}}:=\operatorname{PSH}(X, \omega) \cap \mathcal{C}^{1, \overline{1}}$.

Recall that a $\mathcal{C}^{1, \overline{1}}$-regular function is automatically $\mathcal{C}^{1, a}$-regular for all $0<a<1$. On the other hand, this condition is weaker than $\mathcal{C}^{1,1}$-regularity (i.e. bounded real Hessian).

\subsection{Second-Order Variation of Deligne Functionals}

We have the following identity for the second-order variations of the multivariate energy functional $\langle\cdot, \ldots, \cdot\rangle_{\left(\theta_{0}, \ldots, \theta_{n}\right)}$. 
Proposition 2.8 Let $\theta_{0}, \ldots, \theta_{n}$ be closed $(1,1)$-forms on $X$ and let $\left(\varphi_{i}^{t}\right)_{t \geqslant 0}$ be a smooth ray of smooth functions. Let $\tau:=e^{-t+i s}$ and consider the reparametrised ray $\left(\varphi_{i}^{\tau}\right)_{\tau \in \bar{\Delta}^{*}}$. Denoting by $\Phi_{i}$ the corresponding $S^{1}$-invariant function on $X \times \Delta^{*}$, we have

$$
d d_{\tau}^{c}\left\langle\varphi_{0}^{\tau}, \ldots, \varphi_{n}^{\tau}\right\rangle_{\left(\theta_{0}, \ldots, \theta_{n}\right)}=\int_{X}\left(p_{1}^{*} \theta_{0}+d d_{(x, \tau)}^{c} \Phi_{0}\right) \wedge \cdots \wedge\left(p_{1}^{*} \theta_{n}+d d_{(x, \tau)}^{c} \Phi_{n}\right)
$$

where $\int_{X}$ denotes fibre integration, i.e. pushforward of currents.

Proof The result follows from a computation relying on integration by parts and is an immediate adaptation of, for instance, [7, Proposition 6.2].

As a particular case of the above, we obtain the familiar formulas for the second-order variation of $E$ and $E^{\theta}$, given by

$$
d d_{\tau}^{c} \mathrm{E}\left(\varphi_{\tau}\right)=\frac{1}{n+1} V^{-1} \int_{X}\left(p_{1}^{*} \omega+d d_{(x, \tau)}^{c} \Phi\right)^{n+1}
$$

and

$$
d d_{\tau}^{c} \mathrm{E}^{\theta}\left(\varphi_{\tau}\right)=V^{-1} \int_{X}\left(p_{1}^{*} \omega+d d_{(x, \tau)}^{c} \Phi\right)^{n} \wedge \theta
$$

, respectively. In particular, note that $\mathrm{E}\left(\varphi_{\tau}\right):=\mathrm{E} \circ \Phi$ is a subharmonic function on $\bar{\Delta}^{*}$, whenever $\left(\varphi_{\tau}\right)$ is a subgeodesic. The function $t \mapsto \mathrm{E}\left(\varphi_{\tau}\right)$ is affine along weak geodesics, and convex along subgeodesics.

\subsection{The K-Energy and the Chen-Tian Formula}

Let $\omega$ be a Kähler form on $X$ and consider any path $\left(\varphi_{t}\right)_{t \geqslant 0}$ in the space $\mathcal{H}$ of Kähler potentials on $X$. The Mabuchi functional (or K-energy) $\mathrm{M}: \mathcal{H} \rightarrow \mathbb{R}$ is then defined by its Euler-Lagrange equation

$$
\frac{d}{d t} \mathbf{M}\left(\varphi_{t}\right)=V^{-1} \int_{X} \dot{\varphi}_{t}\left(\mathcal{S}\left(\omega_{\varphi_{t}}\right)-\overline{\mathcal{S}}\right) \omega_{\varphi_{t}}^{n}
$$

It is indeed independent of the path chosen, and the critical points of the Mabuchi functional are precisely the cscK metrics, when they exist. By the Chen-Tian formula [14] it is possible to write the Mabuchi functional as a sum of an "energy" and an "entropy" part. More precisely, with our normalisations we have

$$
\mathrm{M}(\varphi)=\left(\overline{\mathcal{S}} \mathrm{E}(\varphi)-\mathrm{E}^{\operatorname{Ric}(\omega)}(\varphi)\right)+V^{-1} \int_{X} \log \left(\frac{\left(\omega+d d^{c} \varphi\right)^{n}}{\omega^{n}}\right)\left(\omega+d d^{c} \varphi\right)^{n}
$$

where the latter term is the relative entropy of the probability measure $\mu:=\omega_{\varphi}^{n} / V$ with respect to the reference measure $\mu_{0}:=\omega^{n} / V$. Recall that the entropy takes values in $[0,+\infty]$ and is finite if $\mu / \mu_{0}$ is bounded. It can be seen to be always lower semi-continuous (lsc) in $\mu$. 
Following Chen [14] (using the formula (5)) we will often work with the extension $\mathrm{M}: \mathcal{H}^{1, \overline{1}} \rightarrow \mathbb{R}$ of the Mabuchi functional to the space of $\omega$-psh functions with bounded Laplacian. This is a natural setting to consider, since weak geodesic rays with bounded Laplacian are known to always exist, cf. [9, 15,20,21] as well as Lemma 4.6.

For later use, we also state the following definition.

Definition 2.9 The Mabuchi K-energy functional is said to be coercive if there are constants $\delta, C>0$ such that

$$
\mathbf{M}(\varphi) \geqslant \delta \mathbf{J}(\varphi)-C
$$

for all $\varphi \in \mathcal{H}$.

We further recall that the Mabuchi functional is convex along weak geodesic rays, as was recently established by [6], see also [19]. As a consequence of this convexity, the Mabuchi functional is bounded from below (in the given Kähler class) whenever $\alpha$ contains a cscK metric, see [29,35] for a proof in the polarised case and [6] for the general Kähler setting.

\section{Cohomological Test Configurations and K-Semistability}

In this section we introduce a natural generalised notion of test configurations and $\mathrm{K}$-semistability of $(X, \alpha)$ that has meaning even when the class $\alpha \in H^{1,1}(X, \mathbb{R})$ is non-integral (or non-rational), i.e. when $\alpha$ is not necessarily of the form $c_{1}(L)$ for some ample (Q্)-line bundle $L$ on $X$. As remarked by Berman in [4], it is natural to generalise the notion of test configuration in terms of cohomology classes. In the polarised setting, the idea is to consider $\left(\mathcal{X}, c_{1}(\mathcal{L})\right)$ as a "test configuration" for $\left(X, c_{1}(L)\right)$, by simply replacing $\mathcal{L}$ and $L$ with their respective first Chern classes. This approach is motivated in detail below. Moreover, a number of basic and useful properties will be established, and throughout, this generalisation will systematically be compared to the original notion of algebraic test configuration $(\mathcal{X}, \mathcal{L})$ for $(X, L)$, introduced by Donaldson in [27].

Remark 3.1 Much of the following exposition goes through even when the cohomology class $\alpha$ is not Kähler. Unless explicitly stated otherwise, we thus assume that $\alpha=[\theta]$ for some closed $(1,1)$-form $\theta$ on $X$.

\subsection{Test Configurations for $X$}

We first introduce the notion of test configuration $\mathcal{X}$ for $X$, working directly over $\mathbb{P}^{1}$. For the sake of comparison, recall the usual concept of test configuration for polarised manifolds, see, e.g. [12,48]. In what follows, we refer to [31] for background on normal complex spaces.

Definition 3.2 A test configuration $\mathcal{X}$ for $X$ consists of

- a normal compact Kähler complex space $\mathcal{X}$ with a flat morphism $\pi: \mathcal{X} \rightarrow \mathbb{P}^{1}$ 
- a $\mathbb{C}^{*}$-action $\lambda$ on $\mathcal{X}$ lifting the canonical action on $\mathbb{P}^{1}$

- a $\mathbb{C}^{*}$-equivariant isomorphism

$$
\mathcal{X} \backslash \mathcal{X}_{0} \simeq X \times\left(\mathbb{P}^{1} \backslash\{0\}\right)
$$

The isomorphism 6 gives an open embedding of $X \times\left(\mathbb{P}^{1} \backslash\{0\}\right)$ into $\mathcal{X}$, hence induces a canonical $\mathbb{C}^{*}$-equivariant bimeromorphic map $\mu: \mathcal{X} \rightarrow X \times \mathbb{P}^{1}$. We say that $\mathcal{X}$ dominates $X \times \mathbb{P}^{1}$ if the above bimeromorphic map $\mu$ is a morphism. Taking $\mathcal{X}^{\prime}$ to be the normalisation of the graph of $\mathcal{X} \rightarrow X \times \mathbb{P}^{1}$ we obtain a $\mathbb{C}^{*}$-equivariant bimeromorphic morphism $\rho: \mathcal{X}^{\prime} \rightarrow \mathcal{X}$ with $\mathcal{X}^{\prime}$ normal and dominating $X \times \mathbb{P}^{1}$. In the terminology of [12] such a morphism $\rho$ is called a determination of $\mathcal{X}$. In particular, a determination of $\mathcal{X}$ always exists. By the above considerations we will often, up to replacing $\mathcal{X}$ by $\mathcal{X}^{\prime}$, be able to assume that the given test configuration for $X$ dominates $X \times \mathbb{P}^{1}$.

Moreover, any test configuration $\mathcal{X}$ for $X$ can be dominated by a smooth test configuration $\mathcal{X}^{\prime}$ for $X$ (where we may even assume that $\mathcal{X}_{0}^{\prime}$ is a divisor of simple normal crossings). Indeed, by Hironaka (see [33, Theorem 45] for the precise statement concerning normal complex spaces) there is a $\mathbb{C}^{*}$-equivariant proper bimeromorphic map $\mu: \mathcal{X}^{\prime} \rightarrow \mathcal{X}$, with $\mathcal{X}^{\prime}$ smooth, such that $\mathcal{X}_{0}^{\prime}$ has simple normal crossings and $\mu$ is an isomorphism outside of the central fibre $\mathcal{X}_{0}$.

As a further consequence of the isomorphism (6), note that if $\Phi$ is a function on $\mathcal{X}$, then its restriction to each fibre $\mathcal{X}_{\tau} \simeq X, \tau \in \mathbb{P}^{1} \backslash\{0\}$ identifies with a function on $X$. The function $\Phi$ thus gives rise to a family of functions $\left(\varphi_{t}\right)_{t \geqslant 0}$ on $X$, recalling our convention of reparametrising so that $t:=-\log |\tau|$.

Remark 3.3 When $X$ is projective (hence algebraic), the GAGA principle shows that the usual (i.e. algebraic, and normal) test configurations of $X$ correspond precisely to the test configurations (in our sense of Definition 3.2) with $\mathcal{X}$ projective.

\subsection{Cohomological Test Configurations for $(X, \alpha)$}

We now introduce a natural generalisation of the usual notion of algebraic test configuration $(\mathcal{X}, \mathcal{L})$ for a polarised manifold $(X, L)$. This following definition involves the Bott-Chern cohomology on normal complex spaces, i.e. the space of locally $d d^{c}$ exact (1,1)-forms (or currents) modulo globally $d d^{c}$-exact (1,1)-forms (or currents). The Bott-Chern cohomology is finite dimensional and the cohomology classes can be pulled back. Moreover, $H_{\mathrm{BC}}^{1,1}(\mathcal{X}, \mathbb{R})$ coincides with the usual Dolbeault cohomology $H^{1,1}(\mathcal{X}, \mathbb{R})$ whenever $\mathcal{X}$ is smooth. See, e.g. [11] for background.

Definition 3.4 A cohomological test configuration for $(X, \alpha)$ is a pair $(\mathcal{X}, \mathcal{A})$ where $\mathcal{X}$ is a test configuration for $X$ and $\mathcal{A} \in H_{\mathrm{BC}}^{1,1}(\mathcal{X}, \mathbb{R})^{\mathbb{C}^{*}}$ is a $\mathbb{C}^{*}$-invariant $(1,1)$-BottChern cohomology class whose image under the $\mathbb{C}^{*}$-equivariant isomorphism

$$
\mathcal{X} \backslash \mathcal{X}_{0} \simeq X \times\left(\mathbb{P}^{1} \backslash\{0\}\right)
$$

is $p_{1}^{*} \alpha$. Here $p_{1}: X \times \mathbb{P}^{1} \rightarrow X$ is the first projection. 
Definition 3.5 We say that a test configuration $(\mathcal{X}, \mathcal{A})$ for $(X, \alpha)$ is smooth if the total space $\mathcal{X}$ is smooth. In case $\alpha \in H^{1,1}(X, \mathbb{R})$ is $\mathrm{Kähler,} \mathrm{we} \mathrm{say} \mathrm{that}(\mathcal{X}, \mathcal{A})$ is relatively Kähler if the cohomology class $\mathcal{A}$ is relatively Kähler, i.e. there is a Kähler form $\beta$ on $\mathbb{P}^{1}$ such that $\mathcal{A}+\pi^{*} \beta$ is Kähler on $\mathcal{X}$.

Exploiting the discussion following Definition 3.2 we in practice restrict attention to the situation when $(\mathcal{X}, \mathcal{A})$ is a smooth (cohomological) test configuration for $(X, \alpha)$ dominating $X \times \mathbb{P}^{1}$, with $\mu: \mathcal{X} \rightarrow X \times \mathbb{P}^{1}$ the corresponding $\mathbb{C}^{*}$-equivariant bimeromorphic morphism. This situation is studied in detail in Sect. 3.4, where we in particular show that the class $\mathcal{A} \in H^{1,1}(\mathcal{X}, \mathbb{R})$ is always of the form $\mathcal{A}=\mu^{*} p_{1}^{*} \alpha+[D]$ for a unique $\mathbb{R}$-divisor $D$ supported on the central fibre, cf. Proposition 3.10.

It is further natural to ask how the above notion of cohomological test configurations compares to the algebraic test configurations introduced by Donaldson in [27]. On the one hand, we have the following example:

Example 3.6 If $(\mathcal{Y}, \mathcal{L})$ is an algebraic test configuration for $(X, L)$ and we let $\overline{\mathcal{Y}}, \overline{\mathcal{L}}$ and $\bar{L}$, respectively, denote the $\mathbb{C}^{*}$-equivariant compactifications over $\mathbb{P}^{1}$, then $\left(\overline{\mathcal{Y}}, c_{1}(\mathcal{L})\right)$ is a cohomological test configuration for $\left(X, c_{1}(L)\right)$, canonically induced by $(\mathcal{Y}, \mathcal{L})$.

On the other hand, there is no converse such correspondence. For instance, even if $(X, L)$ is a polarised manifold there are more cohomological test configurations $(\mathcal{X}, \mathcal{A})$ for $\left(X, c_{1}(L)\right)$ than algebraic test configurations $(\mathcal{Y}, \mathcal{L})$ for $(X, L)$. However, we show in Proposition 3.14 that such considerations are not an issue in the study of K-semistability of $(X, \alpha)$.

\subsection{The Donaldson-Futaki Invariant and K-Semistability}

The following generalisation of the Donaldson-Futaki invariant is straightforward, at least when the test configuration is smooth (in general one can use resolution of singularities to make sense of the intersection number below).

Definition 3.7 Let $(\mathcal{X}, \mathcal{A})$ be a cohomological test configuration for $(X, \alpha)$. The Donaldson-Futaki invariant of $(\mathcal{X}, \mathcal{A})$ is

$$
\operatorname{DF}(\mathcal{X}, \mathcal{A}):=\frac{\overline{\mathcal{S}}}{n+1} V^{-1}\left(\mathcal{A}^{n+1}\right) \mathcal{X}+V^{-1}\left(K_{\mathcal{X} / \mathbb{P}^{1}} \cdot \mathcal{A}^{n}\right) \mathcal{X}
$$

We recall that $\mathcal{X}$ is assumed to be compact, cf. Definition 3.2, and that $K_{\mathcal{X} / \mathbb{P}^{1}}:=$ $K_{\mathcal{X}}-\pi^{*} K_{\mathbb{P} 1}$ denotes the relative canonical divisor. The point is that by results of Wang [53] and Odaka [39] $\operatorname{DF}\left(\overline{\mathcal{Y}}, c_{1}(\mathcal{L})\right)$ coincides with $\operatorname{DF}(\mathcal{Y}, \mathcal{L})$ whenever $(\mathcal{Y}, \mathcal{L})$ is an algebraic test configuration for a polarised manifold $(X, L)$, with $\mathcal{Y}$ normal (see the proof of Proposition 3.14). Hence the above quantity is a generalisation of the classical Donaldson-Futaki invariant.

The analogue of K-semistability in the context of cohomological test configurations is defined as follows.

Definition 3.8 We say that $(X, \alpha)$ is $K$-semistable if $\operatorname{DF}(\mathcal{X}, \mathcal{A}) \geqslant 0$ for all relatively Kähler test configurations $(\mathcal{X}, \mathcal{A})$ for $(X, \alpha)$. 
Remark 3.9 With the study of K-semistability in mind, we emphasise that the Donaldson-Futaki invariant $\operatorname{DF}(\mathcal{Y}, \mathcal{L})(\mathrm{cf} .[39,53])$ depends only on $\mathcal{Y}$ and $c_{1}(\mathcal{L})$. The notion of cohomological test configuration emphasises this fact.

In order to further motivate the above definitions, we now introduce a number of related concepts and basic properties that will be useful in the sequel.

\subsection{Test Configurations Characterised by $\mathbb{R}$-Divisors}

Recall that if $(\mathcal{X}, \mathcal{L})$ is an algebraic test configuration for a polarised manifold $(X, L)$ that dominates $(X, L) \times \mathbb{C}$, then $\mathcal{L}=\mu^{*} p_{1}^{*} L+D$ for a unique $\mathbb{Q}$-Cartier divisor $D$ supported on $\mathcal{X}_{0}$, see [12]. Similarly, the following result characterises the classes $\mathcal{A}$ associated with smooth and dominating cohomological test configurations, in terms of $\mathbb{R}$-divisors $D$ supported on the central fibre $\mathcal{X}_{0}$.

Proposition 3.10 Let $(\mathcal{X}, \mathcal{A})$ be a smooth cohomological test configuration for $(X, \alpha)$ dominating $X \times \mathbb{P}^{1}$, with $\mu: \mathcal{X} \rightarrow X \times \mathbb{P}^{1}$ the corresponding canonical $\mathbb{C}^{*}$-equivariant bimeromorphic morphism. Then there exists a unique $\mathbb{R}$-divisor $D$ supported on the central fibre $\mathcal{X}_{0}$ such that

$$
\mathcal{A}=\mu^{*} p_{1}^{*} \alpha+[D]
$$

in $H^{1,1}(\mathcal{X}, \mathbb{R})$.

Proof Let $\alpha:=[\omega] \in H^{1,1}(X, \mathbb{R})$. We begin by proving existence: By hypothesis $\mathcal{X}$ dominates $X \times \mathbb{P}^{1}$ via the morphism $\mu$, such that the central fibre decomposes into the strict transform of $X \times\{0\}$ and the $\mu$-exceptional divisor. We write $\mathcal{X}_{0}=\sum_{i} b_{i} E_{i}$, with $E_{i}$ irreducible. Denoting by $[E]$ the cohomology class of $E$ and by $p_{1}: X \times \mathbb{P}^{1} \rightarrow X$ the projection on the first factor, we then have the following formula:

Lemma 3.11 $H^{1,1}(\mathcal{X})=\mu^{*} p_{1}^{*} H^{1,1}(X) \oplus \bigoplus_{i} \mathbb{R}\left[E_{i}\right]$.

Proof of Lemma 3.11 Let $\Theta$ be a closed $(1,1)$-form on $\mathcal{X}$. Then $T:=\Theta-\mu^{*}\left(\mu_{*} \Theta\right)$ is a closed $(1,1)$-current of order 0 supported on $\cup_{i} E_{i}=\operatorname{Exc}(\mu)$. By Demailly's

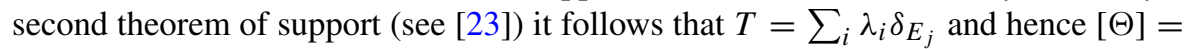
$\mu^{*}\left(\mu_{*}[\Theta]\right)+\sum_{i} \lambda_{i}\left[E_{i}\right]$ in $H^{1,1}(\mathcal{X})$.

Since $H^{1,1}\left(\mathbb{P}^{1}\right)$ is generated by $[0]$, we have $p_{2}^{*} H^{1,1}\left(\mathbb{P}^{1}\right)=\mathbb{R}[X \times\{0\}]$. By the Künneth formula, it thus follows that $H^{1,1}(\mathcal{X})=\mu^{*} p_{1}^{*} H^{1,1}(X) \oplus \mu^{*}(\mathbb{R}[X \times\{0\}]) \oplus$ $\bigoplus_{i} \mathbb{R}\left[E_{i}\right]$.

If we decompose $\mathcal{A}$ accordingly we obtain $\mathcal{A}=\mu^{*} p_{1}^{*} \eta+[D]$ with $D:=\mu^{*}(c[X \times$ $\{0\}])+\bigoplus_{i} b_{i}\left[E_{i}\right]$ and $\eta$ a class in $H^{1,1}(X)$. The restrictions of $\mathcal{A}$ and $\mu^{*} p_{1}^{*} \alpha$ to $\pi^{-1}(1) \simeq X \times\{1\} \simeq X$ are identified with $\alpha$ and $\eta$, respectively. Since $D$ is supported on $\mathcal{X}_{0}$ it follows that $\eta=\alpha$. We thus have the sought decomposition, proving existence.

As for the uniqueness, we let $\mathcal{D}_{0}$ be the set of $\mathbb{R}$-divisors $D$ with support contained in the central fibre $\mathcal{X}_{0}$. Consider the linear map

$$
\begin{aligned}
R: \mathcal{D}_{0} & \rightarrow H^{1,1}(\mathcal{X}) \\
D & \mapsto[D]
\end{aligned}
$$


The desired uniqueness property is equivalent to injectivity of $R$. Hence, assume that $[D]=0$ in $H^{1,1}(\mathcal{X})$. In particular $D_{\mid E_{i}} \equiv 0$ and it follows from a corollary of Zariski's lemma (see, e.g. [1, Lemma 8.2]) that $D=c \mathcal{X}_{0}$, with $c \in \mathbb{R}$. But, letting $\beta$ be any Kähler form on $X$, we see from the projection formula that

$$
\left(\mathcal{X}_{0} \cdot\left(\mu^{*} p_{1}^{*} \beta\right)^{n}\right)_{\mathcal{X}}=\left((X \times\{0\}) \cdot\left(p_{1}^{*} \beta\right)^{n}\right)_{X \times \mathbb{P}^{1}}=\beta^{n}=V>0,
$$

since $V$ is the Kähler volume. Hence $\left[\mathcal{X}_{0}\right]$ is a non-zero class in $H^{1,1}(\mathcal{X})$. It follows that $c=0$, thus $D=0$ as well. We are done.

This gives a very convenient characterisation of smooth cohomological test configurations for $(X, \alpha)$ that dominate $X \times \mathbb{P}^{1}$.

In what follows, we will make use of resolution of singularities to associate a new test configuration $\left(\mathcal{X}^{\prime}, \mathcal{A}^{\prime}\right)$ for $(X, \alpha)$ to a given one, noting that this can be done without changing the Donaldson-Futaki invariant. Indeed, by Hironaka [33, Theorem 45] (see also Sect. 3.2) there is a $\mathbb{C}^{*}$-equivariant proper bimeromorphic map $\mu: \mathcal{X}^{\prime} \rightarrow \mathcal{X}$, with $\mathcal{X}^{\prime}$ smooth and such that $\mathcal{X}_{0}^{\prime}$ has simple normal crossings. Moreover, $\mu$ is an isomorphism outside of the central fibre $\mathcal{X}_{0}$. Set $\mathcal{A}^{\prime}:=\mu^{*} \mathcal{A}$. By the projection formula we then have

$$
\begin{aligned}
\operatorname{DF}\left(\mathcal{X}^{\prime}, \mathcal{A}^{\prime}\right) & =\frac{\overline{\mathcal{S}}}{n+1} V^{-1}\left(\left(\mathcal{A}^{\prime}\right)^{n+1}\right) \mathcal{X}^{\prime}+V^{-1}\left(K_{\mathcal{X}^{\prime} / \mathbb{P}^{1}} \cdot\left(\mathcal{A}^{\prime}\right)^{n}\right) \mathcal{X}^{\prime} \\
& =\frac{\overline{\mathcal{S}}}{n+1} V^{-1}\left(\mathcal{A}^{n+1}\right) \mathcal{X}+V^{-1}\left(K_{\mathcal{X} / \mathbb{P}^{1}} \cdot \mathcal{A}^{n}\right) \mathcal{X}=\operatorname{DF}(\mathcal{X}, \mathcal{A}) .
\end{aligned}
$$

The following result states that it suffices to test K-semistability for a certain class of cohomological test configurations 'characterised by an $\mathbb{R}$-divisor' (in the above sense of Proposition 3.10).

Proposition 3.12 Let $\alpha \in H^{1,1}(X, \mathbb{R})$ be Kähler. Then $(X, \alpha)$ is $K$-semistable if and only if $\operatorname{DF}(\mathcal{X}, \mathcal{A}) \geqslant 0$ for all smooth, relatively Kähler cohomological test configurations $(\mathcal{X}, \mathcal{A})$ for $(X, \alpha)$ dominating $X \times \mathbb{P}^{1}$.

Proof Let $(\mathcal{X}, \mathcal{A})$ be any cohomological test configuration for $(X, \alpha)$ that is relatively Kähler. By Hironaka (see [33]) there is a sequence of blowups $\rho: \mathcal{X}^{\prime} \rightarrow X \times \mathbb{P}^{1}$ with smooth $\mathbb{C}^{*}$-equivariant centres such that $\mathcal{X}^{\prime}$ simultaneously dominates $\mathcal{X}$ and $X \times \mathbb{P}^{1}$ via morphisms $\mu$ and $\rho$, respectively. Moreover, there is a divisor $E$ on $\mathcal{X}^{\prime}$ that is $\rho$ exceptional and $\rho$-ample (and antieffective, i.e. $-E$ is effective). By Proposition 3.10, we have

$$
\mu^{*} \mathcal{A}=\rho^{*} p_{1}^{*} \alpha+[D],
$$

where $D$ is an $\mathbb{R}$-divisor on $\mathcal{X}^{\prime}$ supported on $\mathcal{X}_{0}^{\prime}$. Note that the class $\mu^{*} \mathcal{A} \in$ $H^{1,1}\left(\mathcal{X}^{\prime}, \mathbb{R}\right)$ is relatively nef.

We proceed by perturbation. Since $\alpha$ is Kähler on $X$, we may pick a Kähler class $\eta$ on $\mathbb{P}^{1}$ such that $p_{1}^{*} \alpha+p_{2}^{*} \eta=: \beta$ is Kähler on $X \times \mathbb{P}^{1}$. Since $E$ is $\rho$-ample one may in turn fix an $\varepsilon \in(0,1)$ sufficiently small such that $\rho^{*} \beta+\varepsilon[E]$ is Kähler on $\mathcal{X}^{\prime}$. It follows that $\rho^{*} p_{1}^{*} \alpha+\varepsilon[E]$ is relatively Kähler (with respect to $\mathbb{P}^{1}$ ) on $\mathcal{X}^{\prime}$. Thus $\rho^{*} p_{1}^{*} \alpha+[D]+\delta\left(\rho^{*} p_{1}^{*} \alpha+\varepsilon[E]\right)$ is relatively Kähler for all $\delta \geqslant 0$ small 
enough. In turn, so is $\mathcal{A}_{\delta}^{\prime}:=\rho^{*} p_{1}^{*} \alpha+\left[D_{\delta}\right]$, where $D_{\delta}$ denotes the convex combination $D_{\delta}:=\frac{1}{1+\delta} D+\frac{\delta \varepsilon}{1+\delta} E$. Assuming that the DF-invariant of a smooth and dominating test configuration is always non-negative, it follows from the projection formula and continuity of the Donaldson-Futaki invariant, that

$$
0 \leqslant \operatorname{DF}\left(\mathcal{X}^{\prime}, \mathcal{A}_{\delta}^{\prime}\right) \longrightarrow \operatorname{DF}\left(\mathcal{X}^{\prime}, \mu^{*} \mathcal{A}\right)=\operatorname{DF}(\mathcal{X}, \mathcal{A})
$$

as $\delta \rightarrow 0$. The other direction holds by definition, so this proves the first part of the lemma.

Remark 3.13 With respect to testing K-semistability one can in fact restrict the class of test configurations that need to be considered even further, as explained in Sect. 3.6.

\subsection{Cohomological K-Semistability for Polarised Manifolds}

It is useful to compare cohomological and algebraic K-semistability in the special case of a polarised manifold $(X, L)$.

Proposition 3.14 Let $(X, L)$ be a polarised manifold and let $\alpha:=c_{1}(L)$. Then $\left(X, c_{1}(L)\right)$ is (cohomologically) $K$-semistable if and only if $(X, L)$ is (algebraically) $K$-semistable.

Proof Suppose that $\left(X, c_{1}(L)\right)$ is cohomologically $\mathrm{K}$-semistable. If $(\mathcal{X}, \mathcal{L})$ is an ample test configuration for $(X, L)$, let $\mathcal{A}:=c_{1}(\overline{\mathcal{L}})$. By the intersection theoretic characterisation of the Donaldson-Futaki invariant (Definition 3.7) we then have $\operatorname{DF}(\mathcal{X}, \mathcal{A})=\operatorname{DF}(\mathcal{X}, \mathcal{L}) \geqslant 0$. Hence $(X, L)$ is algebraically $\mathrm{K}$-semistable.

Conversely, suppose that $(X, L)$ is algebraically $\mathrm{K}$-semistable and let $(\mathcal{X}, \mathcal{A})$ be a cohomological test configuration for $(X, \alpha)$. By Lemma 3.12 we may assume that $(\mathcal{X}, \mathcal{A})$ is a smooth, relatively Kähler test configuration for $(X, \alpha)$ dominating $X \times \mathbb{P}^{1}$, with $\mu: \rightarrow X \times \mathbb{P}^{1}$ the corresponding $\mathbb{C}^{*}$-equivariant bimeromorphic morphism. By Proposition 3.10 we further have $\mathcal{A}=\mu^{*} p_{1}^{*} c_{1}(L)+[D]$ for a uniquely determined $\mathbb{R}$-divisor $D$ on $\mathcal{X}$ supported on the central fibre $\mathcal{X}_{0}$. Since $\mathcal{A}$ is relatively Kähler, there is a Kähler form $\eta$ on $\mathbb{P}^{1}$ such that $\mathcal{A}+\pi^{*} \eta$ is Kähler on $\mathcal{X}$. Approximating the coefficients of the divisor $D$ by a sequence of rationals, we write $D=\lim D_{j}$ for $\mathbb{Q}$-divisors $D_{j}$ on $\mathcal{X}$, all supported on $\mathcal{X}_{0}$. As $j \rightarrow+\infty$, we then have

$$
\mu^{*} p_{1}^{*} c_{1}(L)+\left[D_{j}\right]+\pi^{*} \eta \longrightarrow \mathcal{A}+\pi^{*} \eta
$$

which is a Kähler form on $\mathcal{X}$. Since the Kähler cone is open, it follows that $\mu^{*} p_{1}^{*} c_{1}(L)+$ $\left[D_{j}\right]+\pi^{*} \eta$ is also Kähler for all $j$ large enough.

Now let $\mathcal{L}_{j}:=\mu^{*} p_{1}^{*} L+D_{j}$. By the above, $\mathcal{L}_{j}$ is a relatively ample $\mathbb{Q}$-line bundle over $\mathcal{X}$ and $c_{1}\left(\mathcal{L}_{j}\right) \rightarrow \mathcal{A}$. We thus conclude that $\left(\mathcal{X}, \mathcal{L}_{j}\right)$ (for all $j$ large enough) is an ample test configuration for $(X, L)$. Hence

$$
0 \leqslant \mathrm{DF}\left(\mathcal{X}, \mathcal{L}_{j}\right) \longrightarrow \mathrm{DF}(\mathcal{X}, \mathcal{A})
$$

as $j \rightarrow+\infty$, which is what we wanted to prove. 


\subsection{The Non-Archimedean Mabuchi Functional and Base Change}

Let $(\mathcal{X}, \mathcal{A})$ be a cohomological test configuration for $(X, \alpha)$. A natural operation on $(\mathcal{X}, \mathcal{A})$ is that of base change (on $\mathcal{X}$ and we pull back $\mathcal{A}$ ). Unlike resolution of singularities, however, the DF-invariant does not behave well under base change. In this context, a more natural object of study is instead the non-Archimedean Mabuchi functional $\mathrm{M}^{\mathrm{NA}}$ (first introduced in [12,13], where also an explanation of the terminology is given).

Definition 3.15 The non-Archimedean Mabuchi functional is the modification of the Donaldson-Futaki invariant given by

$$
\mathrm{M}^{\mathrm{NA}}(\mathcal{X}, \mathcal{A}):=\operatorname{DF}(\mathcal{X}, \mathcal{A})+V^{-1}\left(\left(\mathcal{X}_{0, \text { red }}-\mathcal{X}_{0}\right) \cdot \mathcal{A}^{n}\right) \mathcal{X}
$$

Note that the 'correction term' $V^{-1}\left(\left(\mathcal{X}_{0, \text { red }}-\mathcal{X}_{0}\right) \cdot \mathcal{A}^{n}\right) \mathcal{X}$ is non-positive and vanishes precisely when the central fibre $\mathcal{X}_{0}$ is reduced. The point of adding to DF this additional term is that the resulting quantity $\mathrm{M}^{\mathrm{NA}}(\mathcal{X}, \mathcal{A})$ becomes homogeneous under base change, i.e. we have the following lemma.

Lemma 3.16 ([12]) Let $(\mathcal{X}, \mathcal{A})$ be a cohomological test configuration for $(X, \alpha)$ and let $d \in \mathbb{N}$. Denote by $\mathcal{X}_{d}$ the normalisation of the base change of $\mathcal{X}$, by $g_{d}: \mathcal{X}_{d} \rightarrow \mathcal{X}$ the corresponding morphism (of degree $d$ ) and set $\mathcal{A}_{d}:=g_{d}^{*} \mathcal{A}$. Then

$$
\mathrm{M}^{\mathrm{NA}}\left(\mathcal{X}_{d}, \mathcal{A}_{d}\right)=d \cdot \mathrm{M}^{\mathrm{NA}}(\mathcal{X}, \mathcal{A})
$$

Proof We refer the reader to [12, Proposition 7.13], whose proof goes through in the analytic case as well.

As an application, it follows from Mumford's semistable reduction theorem ([32, p. 53], see also [34, $\$ 16$, p. 6] for a remark on the analytic case) that there is a $d \in \mathbb{N}$, a finite base change $f: \tau \mapsto \tau^{d}$ (for $d$ 'divisible enough'), a smooth test configuration $\mathcal{X}^{\prime}$ and a diagram

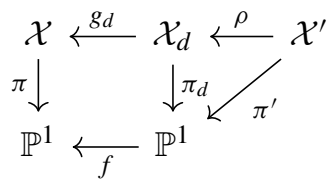

such that $\mathcal{X}^{\prime}$ is semistable, i.e. smooth and such that $\mathcal{X}_{0}^{\prime}$ is a reduced divisor with simple normal crossings. In particular, note that the correction term $V^{-1}\left(\left(\mathcal{X}_{0, \text { red }}^{\prime}-\mathcal{X}_{0}^{\prime}\right) \cdot \mathcal{A}^{n}\right) \mathcal{X}^{\prime}$ vanishes. Here $\mathcal{X}_{d}$ denotes the normalisation of the base change, which is dominated by the semistable test configuration $\mathcal{X}^{\prime}$ for $X$. Moreover, $g_{d} \circ \rho$ is an isomorphism over $\mathbb{P}^{1} \backslash\{0\}$.

Letting $\mathcal{A}_{d}:=g_{d}^{*} \mathcal{A}$ be the pullback of $\mathcal{A}$ to $\mathcal{X}_{d}$, and $\mathcal{A}^{\prime}:=\rho^{*} \mathcal{A}_{d}$ the pullback to $\mathcal{X}^{\prime}$, it follows from the above homogeneity of the $\mathrm{M}^{\mathrm{NA}}$ that

$$
\operatorname{DF}\left(\mathcal{X}^{\prime}, \mathcal{A}^{\prime}\right)=\mathrm{M}^{\mathrm{NA}}\left(\mathcal{X}^{\prime}, \mathcal{A}^{\prime}\right)=\mathrm{M}^{\mathrm{NA}}\left(\mathcal{X}_{d}, \mathcal{A}_{d}\right)=d \cdot \mathrm{M}^{\mathrm{NA}}(\mathcal{X}, \mathcal{A}) \leqslant d \cdot \operatorname{DF}(\mathcal{X}, \mathcal{A}),
$$


where $d$ is the degree of $g_{d}$. We have thus associated with $(\mathcal{X}, \mathcal{A})$ a new test configuration $\left(\mathcal{X}^{\prime}, \mathcal{A}^{\prime}\right)$ for $(X, \alpha)$ such that the total space $\mathcal{X}^{\prime}$ is semistable. Up to replacing $\mathcal{X}^{\prime}$ with a determination (see Sect. 3.1) we can moreover assume that $\mathcal{X}^{\prime}$ dominates $X \times \mathbb{P}^{1}$. Hence, the above shows that $\operatorname{DF}(\mathcal{X}, \mathcal{A}) \geqslant \operatorname{DF}\left(\mathcal{X}^{\prime}, \mathcal{A}^{\prime}\right) / d$. By an argument by perturbation much as the one in the proof of Proposition 3.12, we obtain the following stronger version of the aforementioned result.

Proposition 3.17 Let $\alpha \in H^{1,1}(X, \mathbb{R})$ be Kähler. Then $(X, \alpha)$ is K-semistable (Definition 3.8) if and only if $\operatorname{DF}(\mathcal{X}, \mathcal{A}) \geqslant 0$ for all semistable, relatively Kähler cohomological test configurations $(\mathcal{X}, \mathcal{A})$ for $(X, \alpha)$ dominating $X \times \mathbb{P}^{1}$.

\section{Transcendental Kempf-Ness Type Formulas}

Let $X$ be a compact Kähler manifold of dimension $n$ and let $\theta_{i}, 0 \leqslant i \leqslant n$, be closed $(1,1)$-forms on $X$. Let $\alpha_{i}:=\left[\theta_{i}\right] \in H^{1,1}(X, \mathbb{R})$ be the corresponding cohomology classes. In this section we aim to prove Theorem B. In other words, we establish a Kempf-Ness type formula (for cohomological test configurations), which connects the asymptotic slope of the multivariate energy functional $\left\langle\varphi_{0}^{t}, \ldots, \varphi_{n}^{t}\right\rangle_{\left(\theta_{0}, \ldots, \theta_{n}\right)}$ (see Definition 2.2) with a certain intersection number. In order for such a result to hold, we need to ask that the rays $\left(\varphi_{i}^{t}\right)_{t \geqslant 0}$ are compatible with $\left(\mathcal{X}_{i}, \mathcal{A}_{i}\right)$ in a sense that has to do with extension across the central fibre, see Sect. 4.1.

For what follows, note that, by equivariant resolution of singularities, there is a test configuration $\mathcal{X}$ for $X$ which is smooth and dominates $X \times \mathbb{P}^{1}$. This setup comes with canonical $\mathbb{C}^{*}$-equivariant bimeromorphic maps $\rho_{i}: \mathcal{X} \rightarrow \mathcal{X}_{i}$, respectively. In particular:

Definition 4.1 We define the intersection number

$$
\left(\mathcal{A}_{0} \cdots \mathcal{A}_{n}\right):=\left(\rho_{0}^{*} \mathcal{A}_{0} \cdots \rho_{n}^{*} \mathcal{A}_{n}\right) \mathcal{X}
$$

by means of pulling back the respective cohomology classes to $\mathcal{X}$.

Remark 4.2 Up to desingularising we can and we will in this section consider only smooth cohomological test configurations $\left(\mathcal{X}_{i}, \mathcal{A}_{i}\right)$ for $\left(X, \alpha_{i}\right)$ dominating $X \times \mathbb{P}^{1}$, with $\mu_{i}: \mathcal{X}_{i} \rightarrow X \times \mathbb{P}^{1}$ the corresponding $\mathbb{C}^{*}$-equivariant bimeromorphic morphisms, respectively. We content ourselves by noting that the following $\mathcal{C}^{\infty}$-compatibility condition can be defined (much in the same way, using a desingularisation) in the singular case as well.

\subsection{Compatibility of Rays and Test Configurations}

Let $(\mathcal{X}, \mathcal{A})$ be a smooth (cohomological) test configuration for $(X, \alpha)$ dominating $X \times$ $\mathbb{P}^{1}$, with $\mu: \mathcal{X} \rightarrow X \times \mathbb{P}^{1}$ the corresponding canonical $\mathbb{C}^{*}$-equivariant bimeromorphic morphism. We then have

$$
\mathcal{A}=\mu^{*} p_{1}^{*} \alpha+[D]
$$


for a unique $\mathbb{R}$-divisor $D$ supported on $\mathcal{X}_{0}$, with $p_{1}: X \times \mathbb{P}^{1} \rightarrow X$ denoting the first projection, cf. Proposition 3.10.

We fix the choice of an $S^{1}$-invariant function 'Green function' $\psi_{D}$ for $D$, so that $\delta_{D}=\theta_{D}+d d^{c} \psi_{D}$, with $\theta_{D}$ a smooth $S^{1}$-invariant closed $(1,1)$-form on $\mathcal{X}$. Locally, we thus have

$$
\psi_{D}=\sum_{j} a_{j} \log \left|f_{j}\right| \bmod \mathcal{C}^{\infty},
$$

where (writing $D:=\sum_{j} a_{j} D_{j}$ for the decomposition of $D$ into irreducible components) the $f_{j}$ are local defining equations for the $D_{j}$, respectively. In particular, the choice of $\psi_{D}$ is a uniquely determined modulo smooth function.

The main purpose of this section is to establish Theorem B, which is a formula relating algebraic (intersection theoretic) quantities to asymptotic slopes of Deligne functionals (e.g. E or J) along certain rays. However, such a formula cannot hold for any such ray. The point of the following compatibility conditions is to establish some natural situations in which this formula holds. Technically, recall that a ray $\left(\varphi_{t}\right)_{t} \geqslant 0$ on $X$ is in correspondence with an $S^{1}$-invariant functions $\Phi$ on $X \times \bar{\Delta}^{*}$. The proof of Theorem B, will show that it is important to extend the function $\Phi \circ \mu$ on $\mathcal{X} \backslash \mathcal{X}_{0}$ also across the central fibre $\mathcal{X}_{0}$.

To this end, we introduce the notions of $\mathcal{C}^{\infty}, L^{\infty}$ - and $\mathcal{C}^{1, \overline{1}_{-}}$-compatibility between the ray $\left(\varphi_{t}\right)_{t \geqslant 0}$ and the test configuration $(\mathcal{X}, \mathcal{A})$. The purpose of introducing more than one version of compatibility is that we will distinguish between the following two situations of interest to us:

(i) smooth but not necessarily subgeodesic rays $\left(\varphi_{t}\right)$ that are $\mathcal{C}^{\infty}$-compatible with the smooth test configuration $(\mathcal{X}, \mathcal{A})$ for $(X, \alpha)$, dominating $X \times \mathbb{P}^{1}$. Here we can consider $\alpha=[\theta] \in H^{1,1}(X, \mathbb{R})$ for any closed $(1,1)$-form $\theta$ on $X$.

(ii) locally bounded subgeodesic rays $\left(\varphi_{t}\right)$ that are $L^{\infty}$-compatible or (more restrictively) $\mathcal{C}^{1, \overline{1}}$-compatible with the given smooth and relatively Kähler test configuration $(\mathcal{X}, \mathcal{A})$ for $(X, \alpha)$, dominating $X \times \mathbb{P}^{1}$. Here we thus suppose that $\alpha$ is a Kähler class.

Theorem B has valid formulations in both these situations, as pointed out in Remark 4.11. The second situation is interesting notably with weak geodesic rays in mind, cf. Sect. 4.3.

\section{$4.2 \mathcal{C}^{\infty}$-Compatible Rays}

We first introduce the notion of smooth (not necessarily subgeodesic) rays that are $\mathcal{C}^{\infty}$-compatible with the given test configuration $(\mathcal{X}, \mathcal{A})$ for $(X, \alpha)$.

Definition 4.3 Let $\left(\varphi_{t}\right)_{t} \geqslant 0$ be a smooth ray in $\mathcal{C}^{\infty}(X)$ and denote by $\Phi$ the corresponding smooth $S^{1}$-invariant function on $X \times \bar{\Delta}^{*}$. We say that $\left(\varphi_{t}\right)$ and $(\mathcal{X}, \mathcal{A})$ are $\mathcal{C}^{\infty}$-compatible if $\Phi \circ \mu+\psi_{D}$ extends smoothly across $\mathcal{X}_{0}$.

The condition is indeed independent of the choice of $\psi_{D}$, as the latter is a well-defined modulo smooth function. In the case of a polarised manifold $(X, L)$ with an (algebraic) 
test configuration $(\mathcal{X}, \mathcal{L})$ this condition amounts to demanding that the metric on $\mathcal{L}$ associated with the ray $\left(\varphi_{t}\right)_{t \geqslant 0}$ extends smoothly across the central fibre.

As a useful 'model example' to keep in mind, let $\Omega$ be a smooth $S^{1}$-invariant representative of $\mathcal{A}$ and denote the restrictions $\Omega_{\mid \mathcal{X}_{\tau}}=: \Omega_{\tau}$. Note that $\Omega_{\tau}$ and $\Omega_{1}$ are cohomologous for each $\tau \in \mathbb{P}^{1} \backslash\{0\}$, and hence we may define a ray $\left(\varphi_{t}\right)_{t} \geqslant 0$ on $X, \mathcal{C}^{\infty}$-compatible with $(\mathcal{X}, \mathcal{A})$, by the following relation $\lambda(\tau)^{*} \Omega_{\tau}-\Omega_{1}=d d^{c} \varphi_{\tau}$, where $t=-\log |\tau|$ and $\lambda(\tau): \mathcal{X}_{\tau} \rightarrow \mathcal{X}_{1} \simeq X$ is the isomorphism induced by the $\mathbb{C}^{*}$-action $\lambda$ on $\mathcal{X}$.

We further establish existence of a smooth $\mathcal{C}^{\infty}$-compatible subgeodesic ray associated to a given relatively Kähler test configuration $(\mathcal{X}, \mathcal{A})$ for $(X, \alpha)$.

Lemma 4.4 If $\mathcal{A}$ is relatively Kähler, then $(\mathcal{X}, \mathcal{A})$ is $\mathcal{C}^{\infty}$-compatible with some smooth subgeodesic ray $\left(\varphi_{t}\right)$.

Proof Since $\mathcal{A}$ is relatively Kähler, it admits a smooth $S^{1}$-invariant representative $\Omega$ with $\Omega+\pi^{*} \eta>0$ for some $S^{1}$-invariant Kähler form $\eta$ on $\mathbb{P}^{1}$. By the $d d^{c}$-lemma on $\mathcal{X}$, we have $\Omega=\mu^{*} p_{1}^{*} \omega+\theta_{D}+d d^{c} u$ for some $S^{1}$-invariant $u \in \mathcal{C}^{\infty}(X)$, which may be assumed to be 0 after replacing $\psi_{D}$ with $\psi_{D}-u$. As a result, we get

$$
\Omega=\mu^{*} p_{1}^{*} \omega+\delta_{D}-d d^{c} \psi_{D}
$$

We may also choose a smooth $S^{1}$-invariant function $f$ on a neighbourhood $U$ of $\bar{\Delta}$ such that $\eta_{\mid U}=d d^{c} f$, and a constant $A \gg 1$ such that $D \leqslant A \mathcal{X}_{0}$. Using the Lelong-Poincaré formula $\delta \mathcal{X}_{0}=d d^{c} \log |\tau|$ we get

$$
0<\Omega+\pi^{*} \eta=\mu^{*} p_{1}^{*} \omega+\delta_{D-A \mathcal{X}_{0}}+d d^{c}\left(f \circ \pi+A \log |\tau|-\psi_{D}\right)
$$

on $\pi^{-1}(U)$. Since $D-A \mathcal{X}_{0} \leqslant 0$, it follows that $f \circ \pi+A \log |\tau|-\psi_{D}$ is $\mu^{*} p_{1}^{*} \omega$-psh, and hence descends to an $S^{1}$-invariant $p_{1}^{*} \omega$-psh function $\tilde{\Phi}$ on $X \times U$ (because the fibres of $\mu$ are compact and connected, by Zariski's main theorem). The ray associated with the $S^{1}$-invariant function $\Phi:=\tilde{\Phi}-A \log |\tau|$ has the desired properties.

\section{$4.3 \mathcal{C}^{1, \overline{1}}$-Compatible Rays and the Weak Geodesic Ray Associated with $(\mathcal{X}, \mathcal{A})$}

Let $(\mathcal{X}, \mathcal{A})$ be a smooth, relatively Kähler cohomological test configuration for $(X, \alpha)$ (with $\alpha$ Kähler). With this setup, it is also interesting to consider the following weaker compatibility conditions, referred to as $L^{\infty}$-compatibility and $\mathcal{C}^{1, \overline{1}}$-compatibility, respectively.

Definition 4.5 Let $\left(\varphi_{t}\right)_{t \geqslant 0}$ be a locally bounded subgeodesic ray, and denote by $\Phi$ the corresponding $S^{1}$-invariant locally bounded $p_{1}^{*} \omega$-psh function on $X \times \bar{\Delta}^{*}$. We say that $\left(\varphi_{t}\right)$ and $(\mathcal{X}, \mathcal{A})$ are $L^{\infty}$-compatible if $\Phi \circ \mu+\psi_{D}$ is locally bounded near $\mathcal{X}_{0}$, resp. $\mathcal{C}^{1, \overline{1}}$-compatible if $\Phi \circ \mu+\psi_{D}$ is of class $\mathcal{C}^{1, \overline{1}}$ on $\pi^{-1}(\Delta)$.

Indeed, we will see that $\mathcal{C}^{1, \overline{1}}$-compatibility is always satisfied for weak geodesic rays associated with $(\mathcal{X}, \mathcal{A})$. In particular, for any given test configuration, $\mathcal{C}^{1, \overline{1}}$-compatible 
subgeodesics always exist. This is the content of the following result, which is a consequence of the theory for degenerate Monge-Ampère equations on manifolds with boundary. We refer the reader to [10] for the relevant background.

Lemma 4.6 With the situation (2) in mind, let $(\mathcal{X}, \mathcal{A})$ be a smooth, relatively Kähler cohomological test configuration of $(X, \alpha)$ dominating $X \times \mathbb{P}^{1}$. Then $(\mathcal{X}, \mathcal{A})$ is $\mathcal{C}^{1, \overline{1}}$ compatible with some weak geodesic ray $\left(\varphi_{t}\right)_{t \geqslant 0}$.

Remark 4.7 The proof will show that the constructed ray is actually unique, once a $\varphi_{0} \in \mathcal{H}$ is fixed.

Proof of Lemma 4.6 Let $M:=\pi^{-1}(\bar{\Delta}) \subset \mathcal{X}$. It is a smooth complex manifold with boundary $\partial M=\pi^{-1}\left(S^{1}\right)$.

Let $D, \theta_{D}, \psi_{D}$ and $\Omega$ be as above. Since $\Omega$ is relatively Kähler there is an $\eta \in$ $H^{1,1}\left(\mathbb{P}^{1}\right)$ such that $\Omega+\pi^{*} \eta$ is Kähler on $\mathcal{X}$. We may then write $\tilde{\Omega}=\Omega+\pi^{*} \eta+d d^{c} g$, where $\tilde{\Omega}$ is a Kähler form on $\mathcal{X}$ and $g \in \mathcal{C}^{\infty}(\mathcal{X})$. In a neighbourhood of $\bar{\Delta}$ the form $\eta$ is further $d d^{c}$-exact, and so we write $\eta=d d^{c}\left(g^{\prime} \circ \pi\right)$ for a smooth function $g^{\prime} \circ \pi$ on $\bar{\Delta}$. We now consider the following degenerate complex Monge-Ampère equation:

$$
(\star)\left\{\begin{array}{l}
\left(\tilde{\Omega}+d d^{c} \tilde{\Psi}\right)^{n+1}=0 \text { on } \operatorname{Int}(M) \\
\tilde{\Psi}_{\mid \partial M}=\varphi_{0}+\psi_{D}-g^{\prime}-g
\end{array} .\right.
$$

Since $\tilde{\Omega}$ is Kähler, it follows that there exists a unique $\tilde{\Omega}$-psh function $\tilde{\Psi}$ solving $(\star)$ and that is moreover of class $\mathcal{C}^{1, \overline{1}}$ (see for instance [10, Theorem B]. We now define a $p_{1}^{*} \omega$-psh function on $X \times \bar{\Delta}^{*} \hookrightarrow \mathcal{X}$ by $\mu^{*} \Phi=\tilde{\Psi}-\psi_{D}+g^{\prime}+g$. We then have

$$
\mu^{*}\left(p_{1}^{*} \omega+d d^{c} \Phi\right)=\tilde{\Omega}+d d^{c} \tilde{\Psi}
$$

on $\pi^{-1}\left(\bar{\Delta}^{*}\right)$. In particular, $\Phi$ defines a weak geodesic ray $\left(\varphi_{t}\right)_{t \geqslant 0}$ on $X$. Moreover, the current

$$
\mu^{*} d d^{c} \Phi+\delta_{D}=d d^{c} \tilde{\Psi}+\delta_{D}-d d^{c} \psi_{D}=d d^{c} \tilde{\Psi}+\theta_{D}
$$

has locally bounded coefficients. Indeed, $d d^{c} \tilde{\Psi} \in L_{\text {loc }}^{\infty}$ (as solution of ( $\star$ ), cf. [10]) and $\theta_{D}$ is a smooth $(1,1)$-form on $\overline{\mathcal{X}}$. The constructed ray is thus $\mathcal{C}^{1, \overline{1}}$-compatible with $(\mathcal{X}, \mathcal{A})$.

\subsection{A Useful Lemma}

We now note that in order to compute the asymptotic slope of the Monge-Ampère energy functional $\mathrm{E}$ or its multivariate analogue $\mathrm{E}_{\left(\omega_{0}, \ldots, \omega_{n}\right)}$ we may in fact replace $L^{\infty}$ compatible rays $\left(\varphi^{t}\right)$ with $(\mathcal{X}, \mathcal{A})$ by $\mathcal{C}^{\infty}$-compatible ones. Indeed, note that any two locally bounded subgeodesic rays $\left(\varphi_{t}\right)$ and $\left(\varphi_{t}^{\prime}\right) L^{\infty}$-compatible with $(\mathcal{X}, \mathcal{A})$ satisfy $\Phi \circ \mu=\Phi^{\prime} \circ \mu+O(1)$ near $\mathcal{X}_{0}$, and hence $\varphi_{t}=\varphi_{t}^{\prime}+O(1)$ as $t \rightarrow+\infty$. This leads to the following observation, which will be useful in the view of proving Theorems $\mathrm{B}$ and $\mathrm{C}$. 
Lemma 4.8 Let $\left(\mathcal{X}_{i}, \mathcal{A}_{i}\right)$ be smooth, relatively Kähler cohomological test configurations for $\left(X, \alpha_{i}\right)$, respectively, dominating $X \times \mathbb{P}^{1}$. Let $\left(\varphi_{i}^{t}\right)_{t \geqslant 0}$ and $\left(\varphi_{i}^{\prime t}\right)_{t \geqslant 0}$ be locally bounded subgeodesics that are $L^{\infty}$-compatible with $\left(\mathcal{X}_{i}, \mathcal{A}_{i}\right)$, respectively. Then

$$
\left\langle\varphi_{0}^{t}, \varphi_{1}^{t}, \ldots, \varphi_{n}^{t}\right\rangle_{\left(\omega_{0}, \ldots, \omega_{n}\right)}=\left\langle\varphi_{0}^{\prime t}, \varphi_{1}^{t}, \ldots, \varphi_{n}^{t}\right\rangle_{\left(\omega_{0}, \ldots, \omega_{n}\right)}+O(1)
$$

as $t \rightarrow+\infty$.

Proof For each $i, 0 \leqslant i \leqslant n$, we have $\varphi_{i}^{t}={\varphi^{\prime}}_{i}^{t}+O(1)$ as $t \rightarrow+\infty$. Recall that the mass of the Bedford-Taylor product $\bigwedge\left(\omega_{i}+d d^{c} \varphi_{i}^{t}\right)$ is computed in cohomology, thus independent of $t$. Hence, the quantity

$$
\begin{aligned}
& \left\langle\varphi_{0}^{t}, \varphi_{1}^{t}, \ldots, \varphi_{n}^{t}\right\rangle_{\left(\omega_{0}, \ldots, \omega_{n}\right)}-\left\langle\varphi_{0}^{\prime t}, \varphi_{1}^{t}, \ldots, \varphi_{n}^{t}\right\rangle_{\left(\omega_{0}, \ldots, \omega_{n}\right)} \\
& =\int_{X}\left(\varphi_{0}^{t}-\varphi_{0}^{\prime t}\right)\left(\omega_{1}+d d^{c} \varphi_{1}^{t}\right) \wedge \cdots \wedge\left(\omega_{n}+d d^{c} \varphi_{n}^{t}\right)
\end{aligned}
$$

is bounded as $t \rightarrow+\infty$. By symmetry, the argument may be repeated for the remaining $i$, yielding the result.

\subsection{Asymptotic Slope of Deligne Functionals: Proof of Theorem B}

With the above formalism in place, we are ready to formulate the main result of this section (Theorem B of the introduction). It constitutes the main contribution towards establishing Theorem A, and may be viewed as a transcendental analogue of Lemma 4.3 in [13]. We here formulate and prove the theorem in the 'smooth but not necessarily Kähler' setting (see Sect. 4.1, situation (1)). However, one should note that there is also a valid formulation for $L^{\infty}$-compatible subgeodesics, as pointed out in Remark 4.11 .

Theorem 4.9 Let $X$ be a compact Kähler manifold of dimension $n$ and let $\theta_{i}$, $0 \leqslant i \leqslant n$, be closed $(1,1)$-forms on $X$. Set $\alpha_{i}:=\left[\theta_{i}\right] \in H^{1,1}(X, \mathbb{R})$. Consider smooth cohomological test configurations $\left(\mathcal{X}_{i}, \mathcal{A}_{i}\right)$ for $\left(X, \alpha_{i}\right)$ dominating $X \times \mathbb{P}^{1}$. For each collection of smooth rays $\left(\varphi_{i}^{t}\right)_{t \geqslant 0} \mathcal{C}^{\infty}$-compatible with $\left(\mathcal{X}_{i}, \mathcal{A}_{i}\right)$, respectively, the asymptotic slope of the multivariate energy functional $\langle\cdot, \ldots, \cdot\rangle:=\langle\cdot, \ldots, \cdot\rangle_{\left(\theta_{0}, \ldots, \theta_{n}\right)}$ is well defined and satisfies

$$
\frac{\left\langle\varphi_{0}^{t}, \ldots, \varphi_{n}^{t}\right\rangle}{t} \longrightarrow\left(\mathcal{A}_{0} \ldots \mathcal{A}_{n}\right)
$$

as $t \rightarrow+\infty$. See Sect. 4.1 for the definition of the above intersection number in case the $\mathcal{X}_{i}$ are not all equal.

Proof Fix any smooth $S^{1}$-invariant $(1,1)$-forms $\Omega_{i}$ on $\mathcal{X}_{i}$ such that $\left[\Omega_{i}\right]=\mathcal{A}_{i}$ in $H^{1,1}\left(\mathcal{X}_{i}, \mathbb{R}\right)$. Let $\left(\varphi_{i}^{t}\right)_{t \geqslant 0}$ be smooth and $\mathcal{C}^{\infty}$-compatible with $\left(\mathcal{X}_{i}, \mathcal{A}_{i}\right)$, respectively. Let $\mathcal{X}$ be a smooth test configuration that simultaneously dominates the $\mathcal{X}_{i}$. By pulling 
back to $\mathcal{X}$ we can assume that the $\mathcal{X}_{i}$ are all equal (note that the notion of being $\mathcal{C}^{\infty}$. compatible is preserved under this pullback).

In the notation of Sect. 4.1, the functions $\Phi_{i} \circ \mu+\psi_{D}$ are then smooth on the manifold with boundary $M:=\pi^{-1}(\bar{\Delta})$, and may thus be written as the restriction of smooth $S^{1}$-invariant functions $\Psi_{i}$ on $\mathcal{X}$, respectively.

Using the $\mathbb{C}^{*}$-equivariant isomorphism $\mathcal{X} \backslash \mathcal{X}_{0} \simeq X \times\left(\mathbb{P}^{1} \backslash\{0\}\right)$ we view $\left(\Psi_{i}-\right.$ $\left.\psi_{D}\right)_{\mid \mathcal{X}_{\tau}}$ as a function $\varphi_{i}^{\tau} \in \mathcal{C}^{\infty}(X)$. By Proposition 2.8 we then have

Lemma 4.10 Over $\mathbb{P}^{1} \backslash\{0\}$ we have

$$
d d_{\tau}^{c}\left\langle\varphi_{0}^{t}, \ldots, \varphi_{n}^{t}\right\rangle=\pi_{*}\left(\bigwedge_{i}\left(\Omega_{i}+d d^{c} \Psi_{i}\right)\right) .
$$

Proof The result follows from Proposition 2.8 and the fact that $\mu$ is a biholomorphism away from $\tau=0$, where also $\delta_{D}=0$ (recalling that the $\mathbb{R}$-divisor $D$ is supported on $\mathcal{X}_{0}$ ).

Denoting by $u(\tau):=\left\langle\varphi_{0}^{\tau}, \ldots, \varphi_{n}^{\tau}\right\rangle$, the Green-Riesz formula then yields

$$
\begin{aligned}
\frac{d}{d t}{ }_{t=-\log \varepsilon} u(\tau) & =\int_{\mathbb{P}^{1} \backslash \Delta_{\varepsilon}} d d_{\tau}^{c} u(\tau) \\
& =\int_{\pi^{-1}\left(\mathbb{P}^{1} \backslash \Delta_{\varepsilon}\right)} \bigwedge_{i}\left(\Omega_{i}+d d^{c} \Psi_{i}\right),
\end{aligned}
$$

which converges to $\left(\mathcal{A}_{0} \cdots \mathcal{A}_{n}\right)$ as $\varepsilon \rightarrow 0$.

It remains to show that

$$
\lim _{t \rightarrow+\infty} \frac{u(\tau)}{t}=\lim _{t \rightarrow+\infty} \frac{d}{d t} u(\tau)
$$

To see this, note that for each closed $(1,1)$-form $\Theta$ on $\mathcal{X}$ and each smooth function $\Phi$ on $\mathcal{X}$, there is a Kähler form $\eta$ on $\mathcal{X}$ and a constant $C$ large enough so that $\Theta+C \eta+$ $d d^{c} \Phi \geqslant 0$ on $\mathcal{X}$. Moreover, we have a relation

$$
\left\langle\varphi_{0}^{t}, \varphi_{1}^{t}, \ldots, \varphi_{n}^{t}\right\rangle_{\left(\omega-\omega^{\prime}, \theta_{1} \ldots, \theta_{n}\right)}=\left\langle\varphi_{0}^{t}, \varphi_{1}^{t} \ldots, \varphi_{n}^{t}\right\rangle_{\left(\omega, \theta_{1}, \ldots, \theta_{n}\right)}-\left\langle 0, \varphi_{1}^{t} \ldots, \varphi_{n}^{t}\right\rangle_{\left(\omega^{\prime}, \theta_{1}, \ldots, \theta_{n}\right)}
$$

and repeat this argument for each $i, 0 \leqslant i \leqslant n$, by symmetry. It follows from the above 'multilinearity' that we can write $t \mapsto E\left(\varphi_{0}^{t}, \ldots, \varphi_{n}^{t}\right)$ as a difference of convex functions, concluding the proof.

Remark 4.11 The above proof in fact also yields a version of Theorem 4.9 for $s u b$ geodesics $\left(\varphi_{i}^{t}\right)_{t \geqslant 0}$ that are $L^{\infty}$-compatible with smooth test configurations $\left(\mathcal{X}_{i}, \mathcal{A}_{i}\right)$ for $\left(X, \alpha_{i}\right)$ dominating $X \times \mathbb{P}^{1}$. This follows from the observation that one may replace $L^{\infty}$-compatible subgeodesic rays with smooth $\mathcal{C}^{\infty}$-compatible ones, using Lemmas 4.4 and 4.8 . 
As a special case of Theorem B we obtain transcendental versions of several previously known formulas (see for instance [13]). As an example, we may deduce the following formula for the asymptotics of the Monge-Ampère energy functional by recalling that if $\omega$ is a Kähler form on $X$ and $\left(\varphi_{t}\right)_{t \geqslant 0}$ is a subgeodesic ray, then

$$
\mathrm{E}\left(\varphi_{t}\right)=\frac{1}{(n+1) V}\left\langle\varphi_{t}, \ldots, \varphi_{t}\right\rangle_{(\omega, \ldots, \omega)} .
$$

Corollary 4.12 Assume that $(\mathcal{X}, \mathcal{A})$ is smooth and dominates $X \times \mathbb{P}^{1}$. For each smooth ray $\left(\varphi_{t}\right)_{t \geqslant 0} \mathcal{C}^{\infty}$-compatible with $(\mathcal{X}, \mathcal{A})$, we then have

$$
\lim _{t \rightarrow+\infty} \frac{\mathrm{E}\left(\varphi_{t}\right)}{t}=\mathrm{E}^{\mathrm{NA}}(\mathcal{X}, \mathcal{A})
$$

with

$$
\mathrm{E}^{\mathrm{NA}}(\mathcal{X}, \mathcal{A}):=\frac{\left(\mathcal{A}^{n+1}\right)}{(n+1) V} .
$$

Remark 4.13 Here $\mathrm{E}^{\mathrm{NA}}$ makes reference to the non-Archimedean Monge-Ampère energy functional, see [12] for an explanation of the terminology.

To give a second example of an immediate corollary, interesting in its own right, we state the following (compare [25]):

Corollary 4.14 Assume that $(\mathcal{X}, \mathcal{A})$ is smooth and dominates $X \times \mathbb{P}^{1}$. For each smooth ray $\left(\varphi_{t}\right)_{t \geqslant 0} \mathcal{C}^{\infty}$-compatible with $(\mathcal{X}, \mathcal{A})$, we then have

$$
\lim _{t \rightarrow+\infty} \frac{\mathrm{J}\left(\varphi_{t}\right)}{t}=\mathrm{J}^{\mathrm{NA}}(\mathcal{X}, \mathcal{A})
$$

where

$$
\mathrm{J}^{\mathrm{NA}}(\mathcal{X}, \mathcal{A}):=\frac{\left(\mathcal{A} \cdot \mu^{*} p_{1}^{*} \alpha^{n}\right)}{V}-\mathrm{E}^{\mathrm{NA}}(\mathcal{X}, \mathcal{A})
$$

Proof Note that we may write $\mathrm{J}\left(\varphi_{t}\right)=V^{-1}\left\langle\varphi_{t}, 0, \ldots, 0\right\rangle_{(\omega, \ldots, \omega)}-\mathrm{E}\left(\varphi_{t}\right)$ and apply Theorem 4.9.

\section{Asymptotics for the K-Energy}

Let $(X, \omega)$ be a compact Kähler manifold and $\alpha:=[\omega] \in H^{1,1}(X, \mathbb{R})$ a Kähler class on $X$. As before, let $(\mathcal{X}, \mathcal{A})$ be a smooth, relatively Kähler cohomological test configuration for $(X, \alpha)$ dominating $X \times \mathbb{P}^{1}$. In this section we explain how the above Theorem 4.9 can be used to compute the asymptotic slope of the Mabuchi (K-energy) functional along rays $\left(\varphi^{t}\right), \mathcal{C}^{1, \overline{1}}$-compatible with $(\mathcal{X}, \mathcal{A})$. It is useful to keep the case 
of weak geodesic rays (as constructed in Lemma 4.6) in mind, which in turn implies $\mathrm{K}$-semistability of $(X, \alpha)$ (Theorem A).

Regarding the proof of Theorem $\mathrm{C}$, we will see that the Mabuchi functional is in fact of the form $\left\langle\varphi_{0}^{t}, \ldots, \varphi_{n}^{t}\right\rangle_{\left(\theta_{0}, \ldots, \theta_{n}\right)}$ for the appropriate choice of closed $(1,1)$-forms $\theta_{i}$ on $X$ and rays $\left(\varphi_{i}^{t}\right)$ on $X$, but Theorem 4.9 does not directly apply in this situation. Indeed, the expression for the Mabuchi functional involves the metric $\log \left(\omega+d d^{c} \varphi_{t}\right)^{n}$ on $K_{\mathcal{X} / \mathbb{P}^{1}}$, which may blow up close to $\mathcal{X}_{0}$ (in particular, the compatibility conditions are not satisfied). However, a key point is that we can cook up a functional $\mathbf{M}_{\mathcal{B}}$ of the above 'multivariate' form that satisfies the same asymptotic slope as the Mabuchi functional (up to an explicit error term), and to which we may apply Theorem 4.9. More precisely, we show that

$$
\lim _{t \rightarrow+\infty} \frac{\mathbf{M}\left(\varphi_{t}\right)}{t}=\lim _{t \rightarrow+\infty} \frac{\mathrm{M}_{\mathcal{B}}\left(\varphi_{t}\right)}{t}+V^{-1}\left(\left(\mathcal{X}_{0, \text { red }}-\mathcal{X}_{0}\right) \cdot \mathcal{A}^{n}\right) \mathcal{X},
$$

and use Theorem 4.9 to choose $\mathrm{M}_{\mathcal{B}}$ so that moreover $\lim _{t \rightarrow+\infty} \mathbf{M}_{\mathcal{B}}\left(\varphi_{t}\right) / t=$ $\operatorname{DF}(\mathcal{X}, \mathcal{A})$. It follows that the asymptotic slope of the Mabuchi (K-energy) functional equals $\operatorname{DF}(\mathcal{X}, \mathcal{A})+V^{-1}\left(\left(\mathcal{X}_{0, \text { red }}-\mathcal{X}_{0}\right) \cdot \mathcal{A}^{n}\right) \mathcal{X}=: \mathrm{M}^{\mathrm{NA}}(\mathcal{X}, \mathcal{A})$.

\subsection{A Weak Version of Theorem C}

We first explain how to obtain a weak version of Theorem $\mathrm{C}$, as a direct consequence of Theorem 4.9. This version is more direct to establish than the full Theorem $\mathrm{C}$, and will in fact be sufficient in order to prove $\mathrm{K}$-semistability of $(X, \alpha)$, as explained in Sect. 5.2.

Theorem 5.1 Let $(\mathcal{X}, \mathcal{A})$ be a smooth, relatively Kähler cohomological test configuration for $(X, \alpha)$ dominating $X \times \mathbb{P}^{1}$. For each subgeodesic ray $\left(\varphi^{t}\right)_{t \geqslant 0}$, $\mathcal{C}^{1, \overline{1}}$-compatible with $(\mathcal{X}, \mathcal{A})$, we have the inequality ${ }^{3}$

$$
\varlimsup_{t \rightarrow+\infty} \frac{\mathrm{M}\left(\varphi_{t}\right)}{t} \leqslant \operatorname{DF}(\mathcal{X}, \mathcal{A}) .
$$

Remark 5.2 In view of the strong version (see Theorem 5.6) we actually know that the limit is well defined and, moreover, we obtain this way the precise asymptotic slope of the Mabuchi functional, see Sect. 5.3.

Proof of Theorem 5.1 We use additive notation for line bundles and metrics, meaning that a Hermitian metric $\|$.$\| on a line bundle is L \rightarrow X$ is represented by a collection of local functions $\phi:=\left\{\phi_{U}\right\}$, defined as follows: If $U \subset X$ is an open subset and $s_{U}$ is a trivialising section of $L$ (i.e. a local generator of the invertible sheaf $\mathcal{O}(L)$ ), then we set

$$
\phi_{U}:=-\log \left\|s_{U}\right\|^{2}
$$

\footnotetext{
3 The limit is in fact well defined, as shown in Sect. 5.3.
} 
Here $\phi_{U}$ depends on $s_{U}$, but the curvature current $d d^{c} \phi$ is globally well defined and represents the first Chern class $c_{1}(L)$. In the sequel we identify the additive object $\phi$ with the Hermitian metric it represents.

In the above sense, now let $\mathcal{B}$ be any smooth metric on $K_{\mathcal{X} / \mathbb{P}^{1}}:=K_{\mathcal{X}}-\pi^{*} K_{\mathbb{P}^{1}}$. Consider the canonical isomorphism $\mu: \mathcal{X} \backslash \mathcal{X}_{0} \rightarrow X \times\left(\mathbb{P}^{1} \backslash\{0\}\right)$. Since the restriction of $K_{\mathcal{X} / \mathbb{P}^{1}}$ to each fibre $\mathcal{X}_{t}$ coincides with $K_{\mathcal{X}_{t}}$, which in turn can be identified with $K_{X}$ via $\mu$, we can then associate with $\mathcal{B}$ a ray of smooth metrics on $K_{X}$ that we denote by $\left(\beta_{t}\right)_{t \geqslant 0}\left(\right.$ or $\left(\beta_{\tau}\right)_{\tau \in \bar{\Delta}^{*}}$ for its reparametrisation by $\left.t=-\log |\tau|\right)$. Fix $\log \omega^{n}$ as a reference metric on $K_{X}$, and let

$$
\xi_{\mathcal{B}}^{t}:=\log \left(\frac{e^{\beta_{\tau}}}{\omega^{n}}\right)
$$

, i.e. the function on $X$ given as the difference of metrics $\beta_{\tau}-\log \omega^{n}$ on $K_{X}$. The constructed ray $\left(\xi_{\mathcal{B}}^{t}\right)_{t \geqslant 0}$ is then $\mathcal{C}^{\infty}$-compatible with the cohomological test configuration $\left(\mathcal{X}, K_{\mathcal{X} / \mathbb{P}^{1}}\right)$ for $\left(X, K_{X}\right)$.

Now let $\left(\varphi_{t}\right)_{t \geqslant 0}$ be any subgeodesic ray $\mathcal{C}^{1, \overline{1}}$-compatible with $(\mathcal{X}, \mathcal{A})$. By Lemmas 4.4, 4.8 and Theorem 4.9 it follows that

$$
\frac{\left\langle\xi_{\mathcal{B}}^{t}, \varphi_{t} \ldots, \varphi_{t}\right\rangle_{(-\operatorname{Ric}(\omega), \omega \ldots, \omega)}}{t} \longrightarrow\left(K_{\mathcal{X} / \mathbb{P}^{1}} \cdot \mathcal{A}^{n}\right) \mathcal{X}
$$

as $t \rightarrow+\infty$. Indeed, by Lemma 4.4 we may choose a smooth subgeodesic ray $\left(\varphi_{t}^{\prime}\right)_{t \geqslant 0}$ in $\mathcal{H}$ that is $\mathcal{C}^{\infty}$-compatible (and hence also $L^{\infty}$ - and $\mathcal{C}^{1, \overline{1}}$-compatible) with $(\mathcal{X}, \mathcal{A})$. Up to replacing $\left(\varphi_{t}\right)$ with $\left(\varphi_{t}^{\prime}\right)$ we may thus assume that $\left(\varphi_{t}\right)$ is smooth and $\mathcal{C}^{\infty}$-compatible with $(\mathcal{X}, \mathcal{A})$, using Lemma 4.8 , so that Theorem 4.9 applies.

Motivated by the Chen-Tian formula (5) and the identity (8), we thus introduce the notation

$$
\mathrm{M}_{\mathcal{B}}\left(\varphi_{t}\right):=\overline{\mathcal{S}} \mathrm{E}\left(\varphi_{\tau}\right)+V^{-1}\left\langle\xi_{\mathcal{B}}^{t}, \varphi_{t} \ldots, \varphi_{t}\right\rangle_{(-\operatorname{Ric}(\omega), \omega \ldots, \omega)}
$$

the point being that the asymptotic slope of this functional coincides with the Donaldson-Futaki invariant (even when the central fibre is not reduced).

\section{Lemma 5.3}

$$
\lim _{t \rightarrow+\infty} \frac{\mathrm{M}_{\mathcal{B}}\left(\varphi_{t}\right)}{t}=\operatorname{DF}(\mathcal{X}, \mathcal{A})
$$

Proof This result is an immediate consequence of (8), the Chen-Tian formula (5) and Corollary 4.12.

Hence, it suffices to establish the following inequality

$$
\varlimsup_{t \rightarrow+\infty} \frac{\mathrm{M}\left(\varphi_{t}\right)}{t} \leqslant \lim _{t \rightarrow+\infty} \frac{\mathbf{M}_{\mathcal{B}}\left(\varphi_{t}\right)}{t} .
$$


To do this, we set $\Gamma(\tau):=\left(\mathrm{M}-\mathrm{M}_{\mathcal{B}}\right)\left(\varphi_{t}\right)$. By the Chen-Tian formula (5) and cancellation of terms we have

$$
\begin{aligned}
\Gamma(\tau)= & \overline{\mathcal{S}} \mathrm{E}\left(\varphi_{t}\right)-\mathrm{E}^{\operatorname{Ric}(\omega)}\left(\varphi_{t}\right)+V^{-1} \int_{X} \log \left(\frac{\left(\omega+d d^{c} \varphi_{\tau}\right)^{n}}{\omega^{n}}\right)\left(\omega+d d^{c} \varphi_{\tau}\right)^{n} \\
& -\overline{\mathcal{S}} \mathrm{E}\left(\varphi_{t}\right)-V^{-1}\left\langle\xi_{\mathcal{B}}^{t}, \varphi_{t} \ldots, \varphi_{t}\right\rangle_{(-\operatorname{Ric}(\omega), \omega \ldots, \omega)} \\
= & -\mathrm{E}^{\operatorname{Ric}(\omega)}\left(\varphi_{t}\right)+V^{-1} \int_{X} \log \left(\frac{\left(\omega+d d^{c} \varphi_{\tau}\right)^{n}}{\omega^{n}}\right)\left(\omega+d d^{c} \varphi_{\tau}\right)^{n} \\
& -V^{-1} \int_{X} \xi_{\mathcal{B}}^{t}\left(\omega+d d^{c} \varphi_{\tau}\right)^{n} \\
& +V^{-1} \sum_{j=0}^{n-1} \int_{X} \varphi_{t} \operatorname{Ric}(\omega) \wedge \omega^{j} \wedge\left(\omega+d d^{c} \varphi_{t}\right)^{n-j-1} \\
= & V^{-1} \int_{X} \log \left(\frac{\left(\omega+d d^{c} \varphi_{\tau}\right)^{n}}{\omega^{n}}\right)\left(\omega+d d^{c} \varphi_{\tau}\right)^{n} \\
& -V^{-1} \int_{X} \log \left(\frac{e^{\beta_{\tau}}}{\omega^{n}}\right)\left(\omega+d d^{c} \varphi_{\tau}\right)^{n} \\
= & V^{-1} \int_{X} \log \left(\frac{\left(\omega+d d^{c} \varphi_{\tau}\right)^{n}}{e^{\beta_{\tau}}}\right)\left(\omega+d d^{c} \varphi_{\tau}\right)^{n},
\end{aligned}
$$

recalling the definition (7) of $\xi_{\mathcal{B}}^{t}$ and Definition 2.1.

In view of Proposition 3.10, we as usual let $D$ denote the unique $\mathbb{R}$-divisor supported on $\mathcal{X}_{0}$ such that $\mathcal{A}=\mu^{*} p_{1}^{*} \alpha+[D]$, with $p_{1}: X \times \mathbb{P}^{1} \rightarrow X$ the first projection. Fix a choice of an $S^{1}$-invariant function 'Green function' $\psi_{D}$ for $D$, so that $\delta_{D}=$ $\theta_{D}+d d^{c} \psi_{D}$ with $\theta_{D}$ a smooth $S^{1}$-invariant closed $(1,1)$-form on $\mathcal{X}$. Moreover, set $\Omega:=\mu^{*} p_{1}^{*} \alpha+\theta_{D}$ (for which $[\Omega]=\mathcal{A}$ then holds) and let $\Phi$ denote the $S^{1}$-invariant function on $X \times \mathbb{P}^{1}$ corresponding to the ray $\left(\varphi_{t}\right)$. In particular, the function $\Phi \circ \mu+\psi_{D}$ extends to a smooth $\Omega$-psh function $\Psi$ on $\mathcal{X}$, by $\mathcal{C}^{\infty}$-compatibility.

With the above notation in place, the integrand in the above expression for $\Gamma(\tau)$ can be written as

$$
\log \left(\frac{\left(\omega+d d^{c} \varphi_{\tau}\right)^{n}}{e^{\beta_{\tau}}}\right)=\mu_{*}\left(\log \left(\frac{\left(\Omega+d d^{c} \Psi\right)^{n} \wedge \pi^{*}(\sqrt{-1} d \tau \wedge d \bar{\tau})}{\lambda_{\mathcal{B}}}\right)\right)
$$

where

$$
\lambda_{\mathcal{B}}:=e^{\mathcal{B}+\pi^{*} \log (\sqrt{-1} d \tau \wedge d \bar{\tau})}
$$

is the volume form defined by the smooth metric $\mathcal{B}+\pi^{*} \log (\sqrt{-1} d \tau \wedge d \bar{\tau})$ on $K_{\mathcal{X}}$. Since $\Psi$ is smooth on $\mathcal{X}$ and $\lambda_{\mathcal{B}}$ is a volume form on $\mathcal{X}$, this quantity is bounded from above. Moreover, we integrate against the measure $\left(\omega+d d^{c} \varphi_{\tau}\right)^{n}$ which can be computed in cohomology, and thus has mass independent of $\tau$. Hence 


$$
\Gamma(\tau)=V^{-1} \int_{X} \log \left(\frac{\left(\omega+d d^{c} \varphi_{\tau}\right)^{n}}{e^{\beta_{\tau}}}\right)\left(\omega+d d^{c} \varphi_{\tau}\right)^{n} \leqslant O(1) .
$$

Dividing by $t$ and passing to the limit now concludes the proof.

As explained below, the above 'weak Theorem C' actually suffices to yield our main result.

\subsection{Proof of Theorem A}

We now explain how the above considerations apply to give a proof of Theorem A and point out some immediate and important consequences regarding the YTD conjecture. First recall the following definition (see, e.g. [51, Sect. 7.2]):

Definition 5.4 We say that $(X, \alpha)$ is uniformly $K$-stable if there is a $\delta>0$ and $C \geqslant 0$ such that

$$
\mathrm{M}^{\mathrm{NA}}(\mathcal{X}, \mathcal{A}) \geqslant \delta \mathrm{J}^{\mathrm{NA}}(\mathcal{X}, \mathcal{A})-C
$$

for all relatively Kähler cohomological test configurations $(\mathcal{X}, \mathcal{A})$ for $(X, \alpha)$.

We are now ready to prove Theorem A.

Proof of Theorem $A$ Let $X$ be a compact Kähler manifold and $\omega$ a given Kähler form, with $\alpha:=[\omega] \in H^{1,1}(X, \mathbb{R})$ the corresponding Kähler class. Let $(\mathcal{X}, \mathcal{A})$ be any (possibly singular) cohomological test configuration for $(X, \alpha)$ which by desingularisation and perturbation (see Proposition 3.12) can be assumed to be smooth, relatively Kähler and dominating $X \times \mathbb{P}^{1}$. Consider any ray $\left(\varphi_{t}\right)_{t \geqslant 0}$ such that Theorem $\mathrm{C}$ applies; for instance, one may take $\left(\varphi_{t}\right)$ to be the associated weak geodesic ray emanating from $\omega$ (i.e. such that $\varphi_{0}=0$ ), which due to [15] (cf. also [9,20,21]) is $\mathcal{C}^{1, \overline{1}}$-compatible with $(\mathcal{X}, \mathcal{A})$. Now suppose that the Mabuchi functional is bounded from below (in the given class $\alpha$ ). In particular, we then have

$$
\mathrm{DF}(\mathcal{X}, \mathcal{A}) \geqslant \varlimsup_{\lim _{t \rightarrow+\infty}} \frac{\mathrm{M}\left(\varphi_{t}\right)}{t} \geqslant 0
$$

using the weak version of Theorem $\mathrm{C}$, cf. Theorem 5.1. Since the cohomological test configuration $(\mathcal{X}, \mathcal{A})$ for $(X, \alpha)$ was chosen arbitrarily, this proves Corollary 1.1, i.e. it shows that $(X, \alpha)$ is $\mathrm{K}$-semistable.

In a similar vein, suppose that the Mabuchi functional is coercive, i.e. in particular $\mathbf{M}\left(\varphi_{t}\right) \geqslant \delta \mathbf{J}\left(\varphi_{t}\right)-C$ for some constants $\delta, C>0$ uniform in $t$. Note that Corollary 4.14) and the (weak) Theorem $C$ provides a link with the intersection theoretic quantities $\mathrm{J}^{\mathrm{NA}}(\mathcal{X}, \mathcal{A})$ and $\mathrm{M}^{\mathrm{NA}}(\mathcal{X}, \mathcal{A})$, respectively. More precisely, dividing by $t$ and passing to the limit we have

$$
0 \leqslant \varlimsup_{t \rightarrow+\infty} \frac{(\mathrm{M}-\delta \mathbf{J})\left(\varphi_{t}\right)}{t} \leqslant \mathrm{M}^{\mathrm{NA}}(\mathcal{X}, \mathcal{A})-\delta \mathbf{J}^{\mathrm{NA}}(\mathcal{X}, \mathcal{A})
$$


Since $(\mathcal{X}, \mathcal{A})$ was chosen arbitrarily it follows that $(X, \alpha)$ is uniformly K-stable, concluding the proof of Theorem A.

As remarked in the introduction it follows from convexity of the Mabuchi functional along weak geodesic rays, cf. [6,19], that the Mabuchi functional is bounded from below (in the given class $\alpha$ ) if $\alpha$ contains a cscK representative. In other words, Corollary 1.1 follows.

Moreover, it is shown in [8, Theorem 1.2] that the Mabuchi functional M is in fact coercive if $\alpha$ contains a cscK representative. As a consequence, we obtain also the following stronger result, confirming the "if" direction of the YTD conjecture (here referring to its natural generalisation to the transcendental setting, using the notions introduced in Sect. 3).

Corollary 5.5 If the Kähler class $\alpha \in H^{1,1}(X, \mathbb{R})$ admits a constant scalar curvature representative, then $(X, \alpha)$ is uniformly $K$-stable.

\subsection{Asymptotic Slope of the K-Energy}

Building on Sect. 5.1 we now improve on the weak version of Theorem C (cf. Theorem 5.1) by computing the asymptotic slope of the Mabuchi (K-energy) functional (even when the central fibre is not reduced). To this end, recall the definition of the non-Archimedean Mabuchi functional, i.e. the intersection number

$$
\mathrm{M}^{\mathrm{NA}}(\mathcal{X}, \mathcal{A}):=\operatorname{DF}(\mathcal{X}, \mathcal{A})+V^{-1}\left(\left(\mathcal{X}_{0, \text { red }}-\mathcal{X}_{0}\right) \cdot \mathcal{A}^{n}\right) \mathcal{X}
$$

discussed in Sect. 3.6. Note that it satisfies $\mathrm{M}^{\mathrm{NA}}(\mathcal{X}, \mathcal{A}) \leqslant \operatorname{DF}(\mathcal{X}, \mathcal{A})$ with equality precisely when the central fibre is reduced.

Adapting the techniques of [13] to the present setting we now obtain the following result, corresponding to Theorem $\mathrm{C}$ of the introduction.

Theorem 5.6 Let $X$ be a compact Kähler manifold and $\alpha \in H^{1,1}(X, \mathbb{R})$ a Kähler class. Suppose that $(\mathcal{X}, \mathcal{A})$ is a smooth, relatively Kähler cohomological test configuration for $(X, \alpha)$ dominating $X \times \mathbb{P}^{1}$. Then, for each subgeodesic ray $\left(\varphi_{t}\right)_{t \geqslant 0}$, $\mathcal{C}^{1, \overline{1}}$-compatible with $(\mathcal{X}, \mathcal{A})$, the asymptotic slope of the Mabuchi functional is well defined and satisfies

$$
\frac{\mathrm{M}\left(\varphi_{t}\right)}{t} \longrightarrow \mathrm{M}^{\mathrm{NA}}(\mathcal{X}, \mathcal{A})
$$

as $t \rightarrow+\infty$.

Remark 5.7 In particular, this result holds when $\left(\varphi_{t}\right)_{t \geqslant 0}$ is the weak geodesic ray associated with $(\mathcal{X}, \mathcal{A})$, constructed in Sect. 4.1 .

Proof of Theorem 5.6 Following ideas of [13] we associate with the given smooth, relatively Kähler and dominating test configuration $(\mathcal{X}, \mathcal{A})$ for $(X, \alpha)$ another test configuration $\left(\mathcal{X}^{\prime}, \mathcal{A}^{\prime}\right)$ for $(X, \alpha)$ which is semistable, i.e. smooth and such that $\mathcal{X}_{0}^{\prime}$ is a reduced $\mathbb{R}$-divisor with simple normal crossings. As previously noted, we can also assume that $\mathcal{X}^{\prime}$ dominates the product. In the terminology of Sect. 3.6, this construction 
comes with a morphism $g_{d} \circ \rho: \mathcal{X}^{\prime} \rightarrow \mathcal{X}$, cf. the diagram in Section 3.6. Pulling back, we set $\mathcal{A}^{\prime}:=g_{d}^{*} \rho^{*} \mathcal{A}$. Note that $\mathcal{A}^{\prime}$ is no longer relatively Kähler, but merely relatively semipositive (with the loss of positivity occurring along $\mathcal{X}_{0}^{\prime}$ ).

On the one hand, Lemma 3.16 yields

$$
\mathrm{M}^{\mathrm{NA}}\left(\mathcal{X}^{\prime}, \mathcal{A}^{\prime}\right)=d \cdot \mathrm{M}^{\mathrm{NA}}(\mathcal{X}, \mathcal{A})
$$

where $d>0$ is the degree of the morphism $g_{d}$. On the other hand, we may consider the pullback by $g_{d} \circ \rho$ of the weak geodesic $\left(\varphi_{t}\right)_{t \geqslant 0}$ associated with $(\mathcal{X}, \mathcal{A})$. This induces a subgeodesic $\left(\varphi_{t}^{\prime}\right)_{t \geqslant 0}$ which is $\mathcal{C}^{1, \overline{1}}$-compatible with the test configuration $\left(\mathcal{X}^{\prime}, \mathcal{A}^{\prime}\right)$ for $(X, \alpha)$ (in particular, the boundedness of the Laplacian is preserved under pullback by $\left.g_{d} \circ \rho\right)$. Replacing $\tau$ by $\tau^{d}$ amounts to replacing $t$ by $d \cdot t$, so that

$$
\frac{\mathrm{M}\left(\varphi_{t}^{\prime}\right)}{t}=d \cdot \frac{\mathrm{M}\left(\varphi_{t}\right)}{t}
$$

Combining equations (9) and (10) it thus follows that

$$
\lim _{t \rightarrow+\infty} \frac{\mathrm{M}\left(\varphi_{t}\right)}{t}=\mathrm{M}^{\mathrm{NA}}(\mathcal{X}, \mathcal{A})
$$

if and only if

$$
\lim _{t \rightarrow+\infty} \frac{\mathrm{M}\left(\varphi_{t}^{\prime}\right)}{t}=\operatorname{DF}\left(\mathcal{X}^{\prime}, \mathcal{A}^{\prime}\right)
$$

In other words, it suffices to establish (11). By the asymptotic formula 5.3 it is in turn equivalent to show that

$$
\lim _{t \rightarrow+\infty} \frac{\mathrm{M}\left(\varphi_{t}^{\prime}\right)}{t}=\lim _{t \rightarrow+\infty} \frac{\mathrm{M}_{\mathcal{B}}\left(\varphi_{t}^{\prime}\right)}{t} .
$$

We use the notation of the proof of Theorem 5.1. In particular, we set $\Gamma(\tau):=(\mathrm{M}-$ $\left.\mathrm{M}_{\mathcal{B}}\right)\left(\varphi_{\tau}^{\prime}\right)$. As in the proof of Theorem 5.1 we have an upper bound $\Gamma(\tau) \leqslant O(1)$, using that the restriction of the relatively semipositive class $\mathcal{A}^{\prime}$ to $\mathcal{X}^{\prime} \backslash \mathcal{X}_{0}^{\prime}$ is in fact relatively Kähler.

To obtain a lower estimate of $\Gamma(\tau)$ we consider the Monge-Ampere measure $\operatorname{MA}\left(\varphi_{\tau}^{\prime}\right):=V^{-1}\left(\omega+d d^{c} \varphi_{\tau}^{\prime}\right)^{n}$ and note that

$$
\begin{aligned}
V^{-1} \Gamma(\tau) & =V^{-1} \int_{X} \log \left(\frac{\left(\omega+d d^{c} \varphi_{\tau}^{\prime}\right)^{n}}{e^{\beta_{\tau}}}\right) \\
& =\int_{X} \log \left(\frac{\operatorname{MA}\left(\varphi_{\tau}^{\prime}\right)}{e^{\beta_{\tau}} / \int_{X} e^{\beta_{\tau}}}\right) \operatorname{MA}\left(\varphi_{\tau}^{\prime}\right)-\log \int_{X} e^{\beta_{\tau}} \geqslant-\log \int_{X} e^{\beta_{\tau}},
\end{aligned}
$$

since the relative entropy of the two probability measures $\operatorname{MA}\left(\varphi_{\tau}\right)$ and $e^{\beta_{\tau}} / \int_{X} e^{\beta_{\tau}}$ is non-negative. We now conclude by estimating this integral, using the following result from [13]: 
Lemma 5.8 ([13]). Let $(\mathcal{X}, \mathcal{A})$ be a semistable and dominating test configuration for $(X, \alpha)$ and let $\mathcal{B}$ be any smooth metric on $K_{\mathcal{X} / \mathbb{P}^{1}}$. Let $\left(\beta^{t}\right)_{t \geqslant 0}$ be the family of smooth metrics on $K_{X}$ induced by $\mathcal{B}$. Denote by $p \geqslant 1$ the largest integer such that $p-1$ distinct irreducible components of $\mathcal{X}_{0}$ have a non-empty intersection. Then there are positive constants $A$ and $B$ such that

$$
A t^{2(p-1)} \leqslant \int_{X} e^{\beta^{t}} \leqslant B t^{2(p-1)}
$$

holds for all $t$.

We refer the reader to [13] for the proof and here simply apply the result: Recalling that $t=-\log |\tau|$, Lemma 5.8 yields that $\log \int_{X} e^{\beta_{\tau}}=o(t)$ and so it follows from

$$
\frac{-\log \int_{X} e^{\beta_{\tau}}}{t} \leqslant \frac{\Gamma(\tau)}{t} \leqslant \frac{O(1)}{t}
$$

that

$$
\lim _{t \rightarrow+\infty} \frac{\Gamma(\tau)}{t}=0
$$

completing the proof.

Acknowledgements Since the first version of this paper was made available, R. Dervan and J. Ross, independently, used similar methods to establish Theorem A (see [25]). They are further able to establish K-stability of $(X, \omega)$ whenever the automorphism group $\operatorname{Aut}(X, \omega)$ is discrete. We are grateful to them for helpful discussions on the topic of this paper and related questions. Moreover, it is a pleasure to thank Sébastien Boucksom, Vincent Guedj, Robert Berman and Ahmed Zeriahi, as well as the referees, for helpful discussions and suggestions.

Open Access This article is distributed under the terms of the Creative Commons Attribution 4.0 International License (http://creativecommons.org/licenses/by/4.0/), which permits unrestricted use, distribution, and reproduction in any medium, provided you give appropriate credit to the original author(s) and the source, provide a link to the Creative Commons license, and indicate if changes were made.

\section{References}

1. Barth, W.P., Hulek, K., Peters, C.A.M., Van de Ven, A.: Compact Complex Surfaces, 2nd edn, vol. 4. Ergebnisse der Mathematik und ihrer Grenzgebiete. A Series of Modern Surveys in Mathematics. Springer, Berlin (2004)

2. Bedford, E., Taylor, B.A.: The Dirichlet problem for the complex Monge-Ampère operator. Invent. Math. 37, 1-44 (1976)

3. Bedford, E., Taylor, B.A.: A new capacity for plurisubharmonic functions. Acta Math. 149, 1-40 (1982)

4. Berman, R.: From Monge-Ampère equations to envelopes and geodesic rays in the zero temperature limit. Preprint arXiv: 1307.3008 (2013)

5. Berman, R.: K-polystability of Q-Fano varieties admitting Kähler-Einstein metrics. Invent. Math. 203(3), 1-53 (2016)

6. Berman, R., Berndtsson, B.: Convexity of the K-energy on the space of Kähler metrics and uniqueness of extremal metrics. Preprint arXiv:1405.0401 (2014)

7. Berman, R., Boucksom, S., Guedj, V., Zeriahi, A.: A variational approach to complex Monge-Ampère equations. Publ. Math. de l'IHES 117, 179-245 (2013) 
8. Berman, R., Darvas, T., Lu, C.: Regularity of weak minimizers of the K-energy and applications to properness and K-stability. arXiv:1602.03114v1 (2016)

9. Blocki, Z.: The Calabi-Yau Theorem. Complex Monge-Ampère Equations and Geodesics in the Space of Kähler Metrics, vol. 2038. Springer, Heidelberg (2013)

10. Boucksom, S.: Monge-Ampère equations on complex manifolds with boundary. In: Guedj, V. (ed.) Complex Monge-Ampère Equations and Geodesics in the Space of Kähler Metrics. Lecture Notes in Mathematics, vol. 2038, pp. 257-282. Springer, Berlin (2012)

11. Boucksom, S., Guedj, V.: Regularizing properties of the Kähler-Ricci flow. In: Boucksom, S., Eyssidieux, P., Guedj, V. (eds.) An Introduction to the Kähler-Ricci Flow. Lecture Notes in Mathematics, vol. 2086, pp. 189-238. Springer, Cham (2013)

12. Boucksom, S., Hisamoto, T., Jonsson, M.: Uniform K-stability, Duistermaat-Heckman measures and singularities of pairs. Preprint arXiv:1504.06568v1 (2015)

13. Boucksom, S., Hisamoto, T., Jonsson, M.: Uniform K-stability and asymptotics of energy functionals in Kähler geometry. Preprint arXiv:1603.01026 (2016)

14. Chen, X.X.: On the lower bound of the Mabuchi energy and its application. Int. Math. Res. Not. 12, 607-623 (2000)

15. Chen, X.X.: The space of Kähler metrics. J. Differ. Geom. 56(12), 189-234 (2000)

16. Chen, X.X., Donaldson, S., Sun, S.: Kähler-Einstein metrics on Fano manifolds I: approximation of metrics with cone singularities. J. Am. Math. Soc. 28, 183-197 (2015)

17. Chen, X.X., Donaldson, S., Sun, S.: Kähler-Einstein metrics on Fano manifolds II: limits with cone angle less than 2pi. J. Am. Math. Soc. 28, 199-234 (2015)

18. Chen, X.X., Donaldson, S., Sun, S.: Kähler-Einstein metrics on Fano manifolds III: limits as cone angle approaches 2pi and completion of the main proof. J. Am. Math. Soc. 28, 235-278 (2015)

19. Chen, X.X., Li, L., Paun, M.: Approximation of weak geodesics and subharmonicity of Mabuchi energy. Ann. Fac. Sci. Toulouse Math. 25(5), 935-957 (2016)

20. Darvas, T.: Envelopes and geodesics in spaces of Kähler potentials. Preprint arXiv:1401:7318 (2014)

21. Darvas, T., Lempert, L.: Weak geodesic rays in the space of Kähler metrics. Math. Res. Lett. 19(5), 1127-1135 (2012)

22. Darvas, T., Rubinstein, Y.A.: Tian's properness conjecture and Finsler geometry of the space of Kähler metrics. J. Am. Math. Soc. 30(2), 347-387 (2017)

23. Demailly, J.-P.: Complex Analytic and Differential Geometry. Open source (2012)

24. Dervan, R.: Uniform stability of twisted constant scalar curvature Kähler metrics. Int. Math. Res. Not. 15, 4728-4783 (2016)

25. Dervan, R., Ross, J.: K-stability for Kähler manifolds. Math. Res. Lett. 24(3), 689-739 (2017). doi:10. 4310/MRL.2017.v24.n3.a5

26. Donaldson, S.K.: Anti-self-dual Yang-Mills connections over complex surfaces and stable vector bundles. Proc. Lond. Math. Soc. 50, 1-26 (1985)

27. Donaldson, S.K.: Scalar curvature and stability of toric varieties. J. Differ. Geom 62, 289-349 (2002)

28. Donaldson, S.K.: Lower bounds on the Calabi functional. J. Differ. Geom. 70(3), 453-472 (2005)

29. Donaldson, S.K.: Scalar curvature and projective embeddings. II. Q. J. Math. 56(3), 345-356 (2005)

30. Elkik, R.: Métriques sur les fibrés d'intersection. Duke Math. J. 1, 303-328 (1990)

31. Fischer, G.: Complex Analytic Geometry. Lecture Notes in Mathematics, vol. 538. Springer, Berlin (1976)

32. Kempf, G., Knudsen, F., Mumford, D., Saint-Donat, B.: Toroidal Embeddings 1. Springer, Berlin (1973)

33. Kollár, J.: Lectures on Resolution of Singularities. Annals of Mathematics Studies, vol. 166. Princeton University Press, Princeton (2007)

34. Kollár, J., Nicaise, J., Xu, C.Y.: Semi-stable extensions over 1-dimensional bases. Acta. Math. Sin.English Ser. (2017). doi:10.1007/s10114-017-7048-8

35. Li, C.: Constant scalar curvature Kähler metric obtains the minimum of the K-energy. Int. Math. Res. Not. 9, 2161-2175 (2011)

36. Li, C., Xu, C.: Special test configurations and K-stability of Fano varieties. Ann. Math. 180, 197-232 (2014)

37. Mabuchi, T.: K-stability of constant scalar curvature polarization. arXiv:0812.4093 (2008)

38. Moriwaki, A.: The continuity of Deligne's pairing. Int. Math. Res. Not. 19, 1057-1066 (1999)

39. Odaka, Y.: A generalization of the Ross Thomas slope theory. Osaka J. Math. 50(1), 171-185 (2013)

40. Paul, S., Tian, G.: CM stability and the generalized Futaki invariant I. arXiv: math.AG/0605278 (2006) 
41. Paul, S., Tian, G.: CM stability and the generalized Futaki invariant II. Astérisque 328, 339-354 (2009)

42. Phong, D.H., Ross, J., Sturm, J.: Deligne pairings and the Knudsen-Mumford expansion. J. Differ. Geom. 78(3), 475-496 (2008)

43. Ross, J., Thomas, R.P.: An obstruction to the existence of constant scalar curvature Kähler metrics. J. Differ. Geom. 72, 429-466 (2006)

44. Rubinstein, Y.A.: Smooth and singular Kähler-Einstein metrics. In: Albin, P., et al. (eds.) Geometric and Spectral Analysis. Contemporary Mathematics, pp. 45-138. AMS and Centre Recherches Mathematiques, Montreal (2014)

45. Semmes, S.: Complex Monge-Ampère equations and symplectic manifolds. Am. J. Math 114, 495-550 (1992)

46. Stoppa, J.: K-stability of constant scalar curvature Kähler manifolds. Adv. Math. 221, 1397-1408 (2009)

47. Stoppa, J.: Twisted constant scalar curvature Kähler metrics and Kähler slope stability. J. Differ. Geom. 83, 663-691 (2009)

48. Szekelyhidi, G.: Introduction to extremal Kähler metrics. Graduate Studies in Mathematics, vol. 152. American Mathematical Society, Providence (2014)

49. Tian, G.: Kähler-Einstein metrics with positive scalar curvature. Invent. Math. 130(1), 1-37 (1997)

50. Tian, G.: Bott-Chern forms and geometric stability. Discret. Contin. Dyn. Syst. 6(1), 211-220 (2000)

51. Tian, G.: Canonical Metrics in Kähler Geometry. Lectures in Mathematics ETH Zurich. Birkhauser Verlag, Basel (2000)

52. Tian, G.: K-stability and Kähler-Einstein metrics. Commun. Pure Appl. Math. 68, 1085-1156 (2015)

53. Wang, X.: Height and GIT weight. Math. Res. Lett. 19(04), 909-926 (2012)

54. Yau, S.-T.: Perspectives on geometric analysis. Surv. Differ. Geom. X, 275-379 (2006)

55. Zhang, S.-W.: Heights and reductions of semi-stable varieties. Compos. Math. 104, 77-105 (1996) 